Ann. Abeille, 1966, 9 (4), 27r-32r.

\title{
LE POLLEN DES ERICACEAE DANS LES MIELS FRANÇAIS
}

\author{
G. GENIER \\ Station de Recherches sur l'Abeille et les Insectes sociaux. \\ 91 - Bures-sur-Yvelle
}

SOMMAIRE

L'auteur étudie le pollen de 26 espèces d'Éricacées. L'ornementation du pollen et ses dimensions évaluées statistiquement constituent un bon critère de détermination. Il a été possible d'élaborer une clé de détermination du pollen "frais " des Éricacées. En outre, une partie du travail étudie dans quelle mesure l'aire de distribution mélissologique d'une espèce donnée s'accorde avec celle de distribution géographique.

\section{IN'TRODUC'TION}

Les Ericaceae sensu lato (Vacciniées + Éricacées, BonNrER) représentées en France par une trentaine d'espèces réunies en quelques dix genres, représentent du seul point de vue botanique une famille quantitativement importante. On sait par ailleurs qu'un bon nombre d'espèces sont susceptibles de constituer des peuplements abondants et que la plupart des espèces d'Êricacées sont mellifères, ce qui explique la présence de leur pollen dans un grand nombre de miels français. Or, il se trouve paradoxalement que bien qu'étant rencontrés fréquemment dans les miels, les pollens de cette famille sont encore insuffisamment connus.

Cette constatation nous a amené à envisager l'étude du pollen de toutes les Éricacées de la flore française de façon à aboutir à une description aussi complète que possible des pollens étudiés. Ces pollens devenus identifiables, il nous a semblé bon de les rechercher dans un grand nombre de miels français, afin d'essayer de relier ce que l'on sait de la distribution géographique de la famille aux données relatives à la distribution mélissologique déduites de l'examen des préparations mélissopalynologiques correspondantes. Ce travail devait ultérieurement nous permettre d'estimer l'importance apicole réelle de la famille. 
Einfin, dans une optique quelque peu phytogéographique, nous avons essayé de mettre en évidence des espèces ou groupes d'espèces pouvant être considérés comme caractéristiques d'une région géographique donnée.

\section{I. - LE POLLEN DES ÉRICACÉES}

\section{Io) - GÉNéralités - DÉfinitions}

Chez les Éricacées, le pollen est émis comme chez toutes les dicotylédones gamopétales (quelques espèces gamopétales à ovaire infère telles certaines Campanulacées faisant exception) à l'état trinucléé : la gamétogenèse est donc de type évolué l'anthèse est tardive.

Par ailleurs, le pollen auquel nous avons affaire ici est particulier par le fait qu'il est émis à l'état de tétrade, le cas d'Erica terminalis faisant seul exception. En effet, de façon très générale, les grains formés par quatre dans l'anthère à partir des cellules-mères se dissocient par la suite pour être disséminés isolément : nous parlerons, dans ce cas, d'eumonade (M. Van Campo et Ph. Guine'r, Ig6I). Or, il se trouve que, chez les Éricacées, les quatre cellules-filles issues de la méiose, restent unies tout le temps que dure la gamétogenèse et sont émises sans que la moindre dissociation ne se produise, ce qui donne naissance à une " tétrade".

Une autre particularité de la tétrade des Éricacées réside dans le fait qu'elle est très souvent calymmée, c'est-à-dire que l'ectexine enveloppe l'ensemble des grains de pollen sans présenter d'interruption d'un grain à l'autre (M. VAN CAMPo et $\mathrm{Ph}$. Guinet, I96I); dans le cas contraire, la tétrade est dite acalymmée.
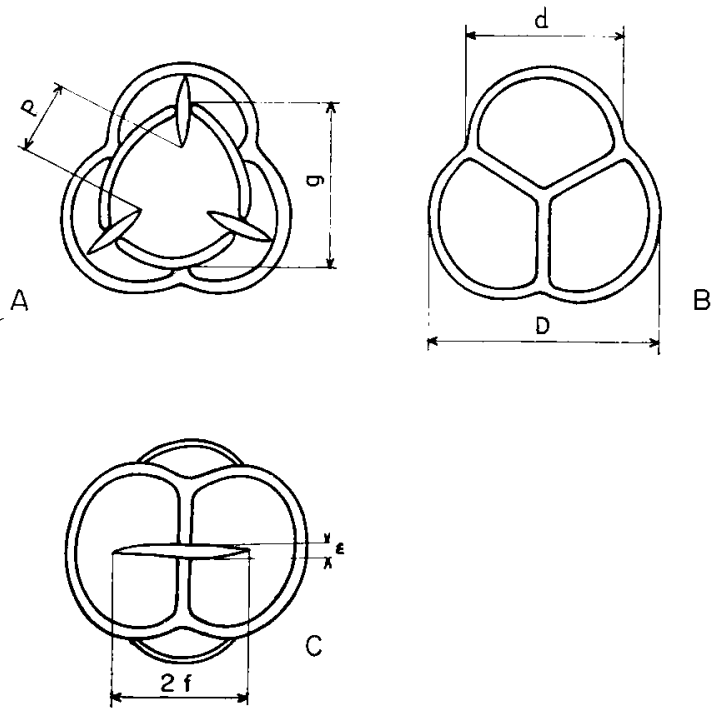

FIG. I. - Vue schématique du pollen en tétrade. Indication des diffirentes mesures effectuées

Sur cette tétrade nous avons mesuré, de même qu'OrDFIELD (I959) l'avait fait sur grains artificiellement fossilisés, les paramètres : $\mathrm{D}, d, g, p$. (fig. I). $2 f$ et $\varepsilon$ représentent la longueur du sillon et la largeur maximum du sillon à l'équateur. 
Les positions intéressantes de la tétrade sont schématisées en " $\mathrm{A}$ ", et " $\mathrm{C}$ "; elles permettent de réaliser toutes les mesures souhaitables et d'analyser exine et sillons grâce à des variations de mise au point.

\section{0) - MÉTHOdẼS D'ÉTUdE}

L'observation des grains est faite à l'aide d'un objectif à immersion, le grossissement obtenu étant de 800 fois.

Toutes les mesures ( $\mathrm{D}, d, g, p, 2 f, \varepsilon)$ portent pour chaque espèce considérée sur 20 grains. A la valeur la plus fréquente obtenue pour chaque mesure, nous ajouterons les valeurs extrêmes trouvées pour $\mathrm{D}, d, g, p$.

\section{$\left.3^{\circ}\right)$ - RÉSULTATS : DESCRIPTION DES ESPÈCES ÉTUDIÉES}

\section{a) Remarques}

Dans le cadre d'une étude des plantes mellifères d'Europe, J. LouvEaux et A. Maurizio (I 964) ont déjà décrit certains pollens d'Éricacées fréquemment rencontrés dans les miels tant suisses que français : il s'agit d'Erica carnea, Erica cinerea, Erica vagans, Calluna vulgaris, Vaccinium myrtillus, Rhododendron ferrugineum. Par ailleurs, F. Ot,DFIEI,D, (I959) s'est déjà préoccupé de l'étude du pollen de cette famille mais cette étude a été réalisée sur pollen fossilisé. Il a ainsi pu observer certains détails particulièrement les endc-cracks, sortes de petites crevasses parcourant l'endexine de la plupart des espèces étudiées et qui ne sont pas visibles sur le pollen frais. De plus, la méthode de l'acétolyse provoque un gonflement des grains ; il s'ensuit que la plupart des dimensions données par OLDFIELD sont supérieures à celles que nous avons obtenues grâce à l'étude du pollen frais. C'est pourquoi nous avons jugé utile de reprendre dans son ensemble l'étude du pollen des Éricacées, dans le but en particulier d'établir une clé de détermination.

\section{b) Description des espèces étudièes (Planches I, 2, et 3)}

Tous les pollens décrits ci-après sont tricolporés.

\section{A - Vacciniées} GILIBERT)

I. Vaccinium myrtillus L. (Vaccinium montanum SALISB. Myrtillus niger " Airelle myrtille".

Symétrie et forme : pollen en tétrade calymmée, régulière, subsphérique, tétrade peu lobée, pôles des grains généralement moins aplatis que ceux du genre Erica.

Dimensions en $\mu$ :

$$
\begin{array}{ll}
\mathrm{D}=35 & (32,5<\mathrm{D}<38,5) \\
d=25 & (22<d<29,3) \\
g=25,7 & (23,5<g<27,5) \\
p=8,4 & (6,2<p<10,8)
\end{array}
$$





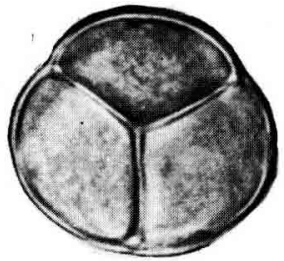

1
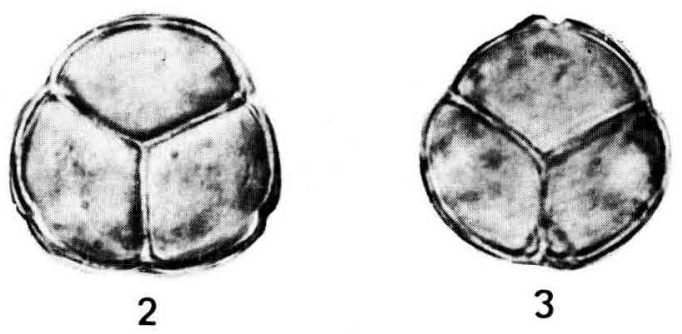

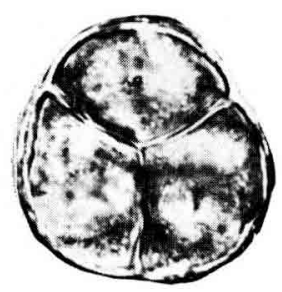

4

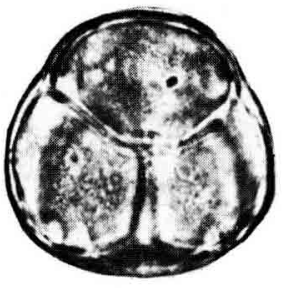

5

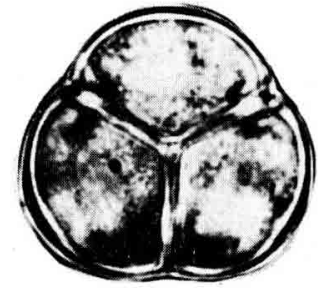

6

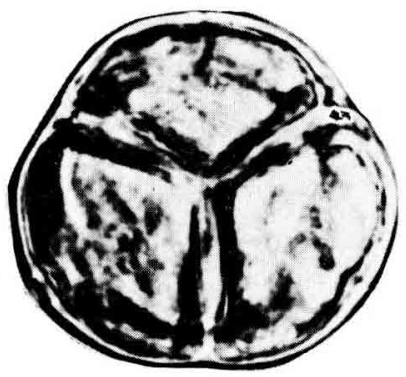

7

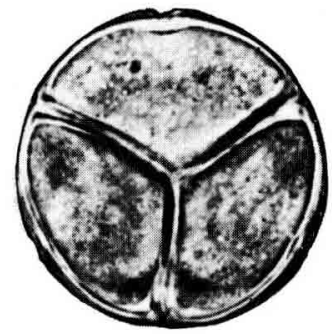

8

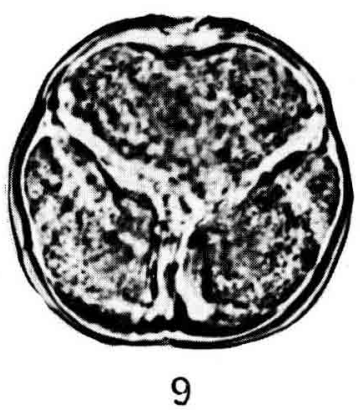

P'LANCHE I

Grossissement 750 : I

I. Vaccinimm myrlillus L. Coupe optique de la tétrade en position $\mathbf{B}$.

2. Vaccinium uliginosum. Coupe optique de la tétrade.

3. Vaccinium vilis idaen. Coupe optique de la tétrade.

4. Oxycoccos vulgaris PuRSH. Coupe optique de la tétrade.

5. Rhododendron ferrugineum. Coupe optique de la tétrade.

6. Rhodotendron hirsulum L. Coupe optique de la tétrade.

7. Arbulus unedo L. Coupe optique de la tétrade.

8. Arbulus uía-msi L. Coupe optique de la tétrarle.

9. Arbulus alpina L. Coupe optique de la titrade. 


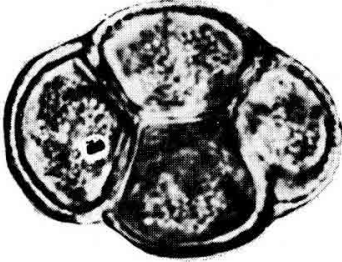

10

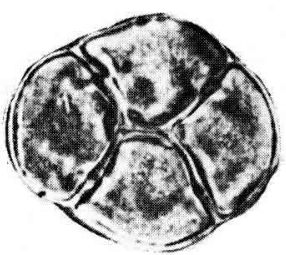

13

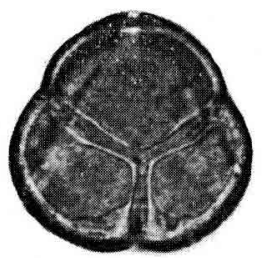

15

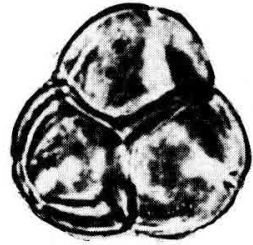

11

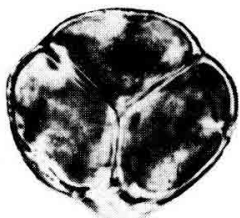

$13 \mathrm{~b}$

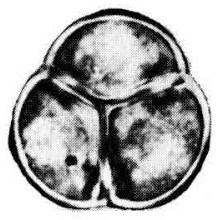

12
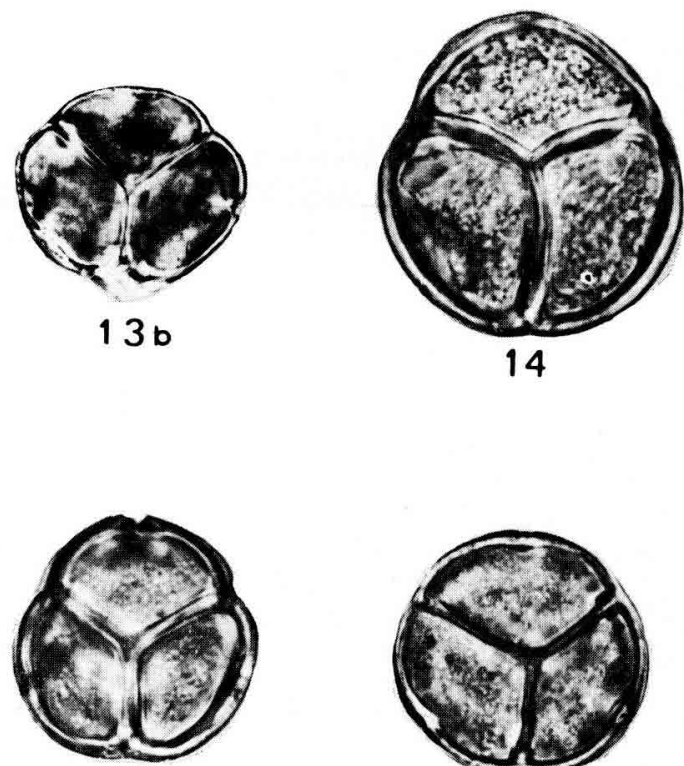

16

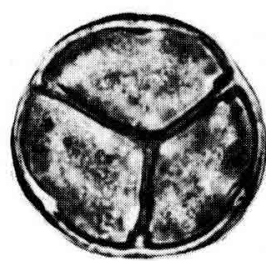

17

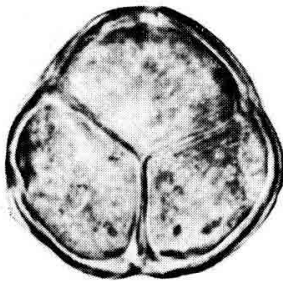

18

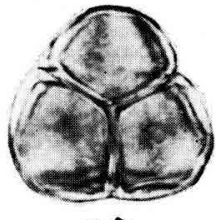

19

Planche 2

Grossissement $75^{\circ}: \mathbf{I}$

Io. Calluna vulgaris. SALIsB. Aspect de la tétrade irrégulière.

I. Daboecia polijolia. Don. Coupe optique de la tétrade en position B.

I2. Loisenleria procumbens. DESV. Coupe optique de la tétrade en position B.

I3 Phyllodoce cerulea. Fries. Tétrade "irrégulière " présentation de 4 grains dans un même plan.

I3 bis. Phyllodoce cenulea. Fries. Coupe optique de la tétrade en position 3.

1. Andromeda polifolia L. Coupe optique de la tétrade assez irrégulière en position B.

15. Erica arborea L. Coupe optique de la tétrade en position B. Noter l'épaisseur des murs internes de la tétrade.

6. Erica scoparia I. Coupe optique de la tétrade en position B. Noter l'épaisseur des murs internes de la tétrade.

17. Erica carnea L. Coupe optique de la tétrade en position B.

18. Evica tetralix L. Coupe optique de la tétrade en position B.

I9. Erica vagans. Coupe optique de la tétrade en position B. Forme sub - triangulaire, pôles des grains très aplatis. 

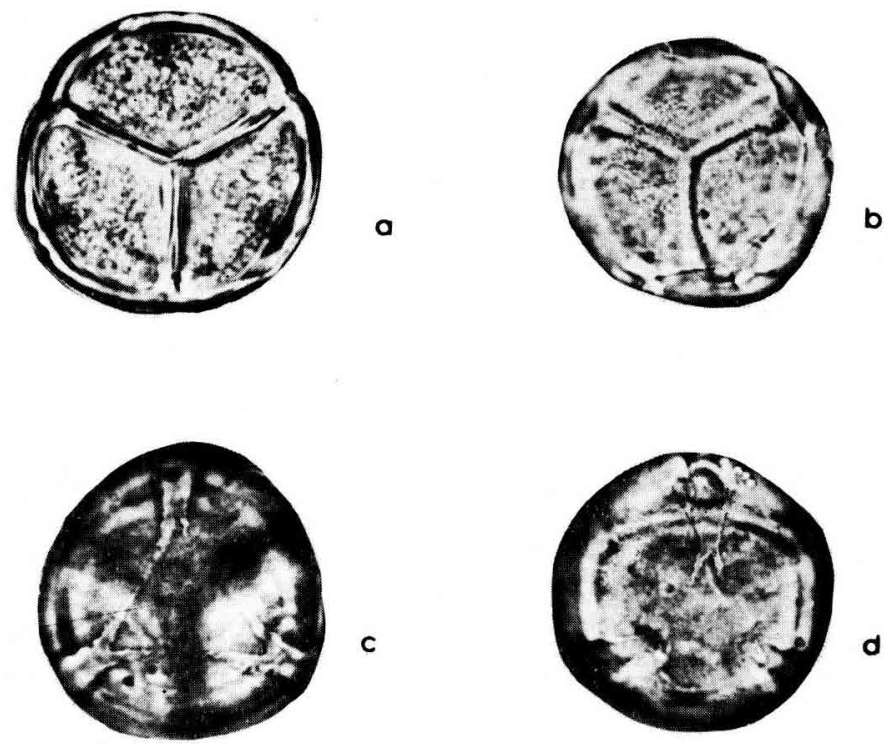

20

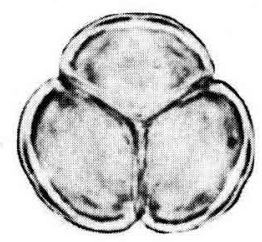

21

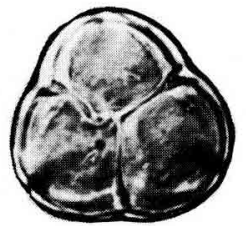

24
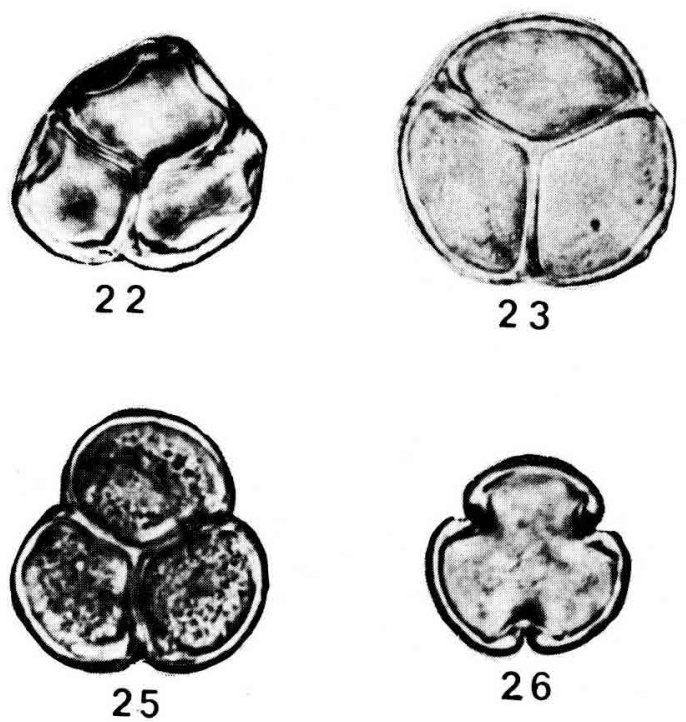

Grossissement 750 : I

20. Erica cinerea L. :

a) Coupe optique de la tétrade en position $B$.

b) Ornementation de l'exine. Mise en évidence du réticulum.

c) Triangle polaire, terminaison bifide des sillons.

d) Le sillon et sa terminaison, le pore, mise au point faite sur le grain supérieur de la tétrade. 21. Evica multiftora I. Coupe optique de la tétrade en position B.

22. Erica lusilanica RUD. Coupe optique de la tétrade en position B.

23. Erica ciliaris I. Coupe optique de la tétrade en position B.

24. Erica mediterranea L. Coupe optique de la tétrade en position B.

25. Evica umbellata $\mathrm{L}$. Coupe optique de la tétrade en position B.

26. Erica siricta Don. Vue équatoriale du grain. 
$\because$. 
Apertures : 6 paires de demi-sillons $\left({ }^{1}\right)$ de largeur maximum. à l'équateur $\varepsilon=3,8 \mu$, bords des sillons irréguliers sans épaississements notables.

Ces sillons se prolongent parfois jusqu'aux pôles des grains ou presque.

Les pores sont petits, circulaires, ou légèrement allongés. I pore par sillon le plus souvent difficilement visible.

Marges : épaississements de l'endexine très peu prononcés.

Exine : mince : I,5 $\mu$ d'épaisseur environ, endexine légèrement plus épaisse que l'ectexine, exine intectée, réticulée, mailles du réticulum régulières sur toute la surface du grain rappelant celles d'Erica ciliaris.

Intine : légèrement moins épaisse que l'ensemble endexine-ectexine.

Cytoplasme peu et finement granuleux.

\section{Vaccinicium uliginosum L. (Vaccinium gaultheroides BIGEL)}

"Airelle du marais ».

Symétrie et forme : tétrađe peu lobée calymmée subsphérique.

Dimensions :

$$
\begin{array}{ll}
\mathrm{D}=38 \mu & (36 \mu<\mathrm{D}<40,5 \mu) \\
d=28,7 \mu & (26,6 \mu<d<30 \mu) \\
g=28,7 \mu & (25,8 \mu<g<30,5 \mu) \\
p=7,2 \mu & (6,1 \mu<p<8,5 \mu)
\end{array}
$$

Apertures : 6 paires de demi-sillons.

Sillons allongés terminés en pointe pouvant atteindre r $9 \mu$ de long.

Pores circulaires, diamètre compris entre $3,5 \mu$ et $4, \mathrm{I} \mu$ et assez éloignés du point de jonction de chaque gain constitutif de la tétrade. I pore par sillon.

Marges peu développées à absentes.

Exine : épaisseur : I,7 $u$ environ, endexine plus épaisse que l'ectexine; ornementation semblable à celle de $V$. myrtillus.

Intine : légèrement plus mince que l'exine.

Cytoplasme : clair, finement granuleux.

3. Vaccinium vitis-idaea L. (Vaccinium punctatum LAM. Vaccinium punctifolium (STOCKES)

"Airelle vigne-du-Mont-Ida ».

Symétrie et forme : pollen en tétrade calymmée présentant une tendance fréquente

à l'irrégularité. Pôles des grains assez peu aplatis.

Dimensions en $\mu$ :

$$
\begin{array}{ll}
\mathrm{D}=35 & (32,6<\mathrm{D}<37,8) \\
d=26 & (24<d<28) \\
g=26 & (23,8<g<28,2) \\
p=\mathrm{I} 2,8 & (9<p<\mathrm{I} 5,2)
\end{array}
$$

(1) Nomenclature de Maurizio et Louveaux ( 1964 ). Nous parlerons en ce qui concerne le paramètre " $2 f$ " de "longueur du sillon ", il s'agit donc en fait de la somme des deux longueurs des deux demi-sillons situés dans le prolongement l'un de l'autre. 
Apertures : 6 paires de demi-sillons.

Sillons plus courts que dans les deux espèces précédentes $(2 f=\mathrm{I} 7 \mu)$ et larges $(5,8 \mu<\varepsilon<6,4 \mu)$ sillons médians à peu près rectilignes pointus mais non effilés à l'extrémité.

Pores : peuvent faire défaut, ellipsoïdaux lorsqu'ils existent, pouvant atteindre $5,5 \mu$ de large.

Marges assez marquées et caractérisées par leur faible extension latérale.

Exine : reticulum de l'exine généralement plus net et à mailles plus larges que chez les autres V'raccinium, endexine plus épaisse que l'ectexine, l'ensemble atteignant $\mathrm{I}, 7 \mu$ d'épaisseur.

Intine : légèrement plus mince que l'exine.

Cytoplasme : clair, pet granuleux.

4. Oxycoccos vulgaris PURSH. (Vaccinium oxycoccos L. Oxycoccos palustris PERS. Oxycoccos quadripetala GILIBERT. Oxycoccos microcarpa TURCZ. Vaccirium palustris SAIJSB. Vaccinium microcarpum Hook).

"Canneberge vulgaire".

Symétrie et forme : pollen en tétrade calymmée, pôles des grains peu aplatis, aspect " frippé " fréquent.

Dimensions en $\mu$ : particulièrement variables.

$$
\begin{array}{ll}
\mathrm{D}=36,2 & (27,2<\mathrm{D}<37,3) \\
d=26 & (24<d<27,6) \\
g=25,9 & (24<g<27,5) \\
p=7,7 & (5,2<p<9,4)
\end{array}
$$

Apertures : 6 paires de demi-sillons.

Sillons allongés $(2 f$ atteint $20,5 \mu$.) étroits difficilement visibles dans quelques cas fourchus à l'extrémité.

Pores elliptiques, relativement étroits, souvent absents. I pore par sillon.

Marges : bien visibles, souvent recouvertes par l'ornementation de l'exine, peu épaisses mais à forte extension latérale (extension pouvant atteindre $4,5 \mu$ ).

Exine : réticulum à mailles nettement plus larges à l'équateur des grains qu'aux pôles, épaisseur de l'exine sensiblement égale à celle de l'ectexine.

Intine : claire et légèrement plus mince que l'exine.

Cytoplasme : granuleux.

$$
\text { B. - Éricacées }
$$

5. Rhododendron ferrugineum L.

"Rhododendron ferrugineux ".

Symétrie et forme : pollen en tétrade calymmée subsphérique : murs internes de la tétrade épais. 
Dimensions (en $\mu$ ) :

$$
\begin{array}{ll}
\mathrm{D}=3^{8} & (35<\mathrm{D}<40,6) \\
d=27 & (25<d<29) \\
g=25 & (24<g<26,3) \\
p=\mathrm{I} 2,5 & (\mathrm{II}<p<\mathrm{I} 3,8)
\end{array}
$$

Apertures : 6 paires de demi-sillons.

Sillons médians, bords nets, à terminaison pointue, quelquefois légèrement bifides.

Largeur maximum du sillon à l'équateur $\varepsilon=4 \mu$.

Longueur maximum du sillon $2 f=\mathrm{I}_{4} \mu$.

Marges : bien visibles atteignant 2,3 $\mu$ d'épaisseur.

Pores : sub-circulaires à contours assez irréguliers, situés sur les sillons, près du point de jonction des grains constituant la tétrade.

Exine : ensemble endexine-ectexine atteignant I,9 $\mu$ d'épaisseur; ectexine lisse s'amincissant à la jonction des grains de la tétrade tandis qu'elle s'épaissit aux pôles de chaque grain. Exine verrulée, éléments de sculpture très petits.

Intine : beaucoup plus mince que l'ensemble ectexine-endexine.

Cytoplasme : clair, non granuleux.

\section{Rhododendron hirsutum $\mathrm{L}$.}

"Rhododendron hérissé "

Forme et symétrie : pollen en tétrade calymmée sub-sphérique légèrement moins globuleuse que dans le cas précédent, murs internes de la tétrade épais $(\mathrm{I}, 5 \mu$ d'épaisseur).

Dimensions (en $\mu$ ) :

$$
\begin{array}{ll}
\mathrm{D}=40 & (37,4<\mathrm{D}<42,2) \\
d=29 & (27<d<3 \mathrm{I}, 3) \\
g=3 \mathrm{I} & (28,2<g<33) \\
p=\mathrm{I} 4,6 & (\mathrm{I} 2,2<p<\mathrm{I} 6,5)
\end{array}
$$

Apertures : 6 paires de demi-sillons.

Sillons courts, médians, larges $(\varepsilon=4,6 \mu)$, terminaisons pointues parfois émoussées, rarement bifides.

Marges : bien visibles bien que peu importantes (ne dépassant pas $2,4 \mu$ d'épaisseur).

Pores clairs et elliptiques à sub-circulaires.

Exine : ensemble ectexine-endexine atteignant $2 \mu$ d'épaisseur. Ectexine s'épaississant aux pôles de chaque grain. Réticulum à mailles extrêtmement fines difficilement visibles.

Intine : plus mince que l'ensemble ectexine-endexine ne dépassant pas I, $2 \mu$ environ. Cytoplasme : clair, non granuleux. 
7. Arbutus unedo L. (Unedo edulis $\mathrm{HG}$ et $\mathrm{I}, \mathrm{K}$ ). "Arbousier unedo".

Forme et symétrie : pollen en tétrade calymmée très globuleuse.

Dimensions en $\mu$ :

$$
\begin{array}{ll}
\mathrm{D}=52,2 & (49<\mathrm{D}<54) \\
d=39,7 & (38<d<4 \mathrm{I}) \\
g=3^{8,6} & (37<g<40,8) \\
p=\mathrm{II} & (9<p<\text { I } 4,3)
\end{array}
$$

Apertures : 6 paires de demi-sillons.

Sillons très longs $(27,2 \mu$ de long) et larges ( $\varepsilon$ atteignant $4,8 \mu)$, pointus aux extrémités, bords des sillons nets.

Marges bien visibles, épaisseur atteignant $3,2 \mu$.

Pores : diamètre d'environ $4,8 \mu$ légèrement ovale à contour souvent irrégulier.

Exine : légèrement granuleuse, endexine plus épaisse que l'ectexine, l'ensemble atteignant $\mathrm{I}, 6 \mu$.

Intine : mince.

Cytoplasme : clair et très finement granuleux.

8. Arbutus uva-ursi L. (Arctostaphylos uva-ursi sPRENG.). (Arctostaphylos officinalis WIMM. et GRAB.).

"Arbousier raisin-d'ours ".

Forme et symétrie : pollen en tétrade calymmée, sphérique, très rarement irrégulière. Dimensions en $\mu$ :

$$
\begin{array}{ll}
\mathrm{D}=43,5 & (39<\mathrm{D}<46,8) \\
d=34 & (3 \mathrm{I}, 9<d<36,7) \\
g=34,5 & \left(32,3<g<3^{6,5)}\right. \\
p=9,2 & (6,5<p<\mathrm{I} 2,3)
\end{array}
$$

donc dimensions assez variables.

Apertures : 6 paires de demi-sillons.

Sillons longs ( $2 f$ atteignant $24,3 \mu$ ) et plus étroits que dans le cas précédent $(\varepsilon=2,8 \mu)$, extrémités des sillons parfois bifides, le plus souvent effilées.

Pores : sub-circulaires aux contours peu visibles, diamètre $: 2,5$ à $2,7 \mu$.

Marges : épaisseur atteignant 2 à $2,3 \mu$, extension latérale variable.

Exine : endexine plus épaisse que l'ectexine, l'ensemble atteignant 2,3 $\mu$ d'épaisseur.

Ornementation verrulée bien visible, les protubérances tendant à se souder par endroit dans certains grains pour constituer un " réticulum " à mailles très irrégulières.

Intine : plus mince que l'exine, I,8 à $2 \mu$ d'épaisseur.

Cytoplasme : granuleux. 
9. Arbutus alpina L. (Arctos alpina HUI,L.). (Arctostaphylos alpina SPRENG.). (Uva-ursi alpina GRAY.).

"Arbousier des Alpes".

Forme et symétrie : pollen en tétrade calymmée, globuleuse, parfois légèrement irrégulière.

Dimensions en $\mu$ :

$$
\begin{array}{ll}
\mathrm{I}=4^{8} & (46,8<\mathrm{D}<50,2) \\
d=3^{8,4} & \left(3^{2}<d<4 \mathrm{I}\right) \\
g=37,8 & (34<g<40) \\
p=\mathrm{I} 0,2 & (8<p<\mathrm{I} 3)
\end{array}
$$

Apertures : 6 paires de demi-sillons.

Sillons longs et étroits $(2 f=2 \mathrm{I} \mu, \varepsilon=\mathrm{I}, 9 \mu)$, terminaisons variables parfois très effilées, parfois "émoussées ", dans certains cas très légèrement bifides, bords des sillons nets.

Pores sub-circulaires, font fréquemment défaut.

Marges peu importantes et recouvertes par l'ectexine.

Exine : endexine moins épaisse que l'ectexine, ornementation verrulée, rugulée, très variable en importance.

Intine : claire, moins épaisse que l'ensemble endexine-ectexine.

Cytoplasme : granuleux clair.

Io. Calluna vulgaris SALISB. (Erica vulgaris L. Calluna erica D. C). "Callune vulgaire $).$

Forme et symétrie : pollen en tétrade calymmée, très souvent fortement déformée Dimensions en $\mu$ :

$$
\begin{array}{ll}
\mathrm{D}=35 & (3 \mathrm{I}, 2<\mathrm{I})<38,3)\left({ }^{1}\right) \\
d=2 \mathrm{I} & (\mathrm{I} 9<d<23,4) \\
g=22 & (20<g<23,7) \\
p=\text { non mesuré } &
\end{array}
$$

Apertures : sillons très difficilement visibles sur la totalité de leur parcours, recouverts par l'ectexine, extrémités effilées.

Marges peu importantes.

Pores : rarement visibles, diamètre d'environ 2,9 à $3 \mu$ (mesuré sur 4 grains seulement) situés près de la ligne de contact de deux grains adjacents.

Exine : épaisseur de l'ensemble ectexine-endexine variant de $2, \mathrm{I}$ à $2,7 \mu$, épaisseur de l'endexine sensiblement égale à celle de l'ectexine; exine tectée, columelles bifides à leur base particulièrement visibles typiques du genre Calluma. Le tectum porte un réticulum dont les mailles ont des dimensions très variables.

Intine : mince.

Cytoplasme : peu clair, légèrement granuleux.

(1) D est mesuré sur des tétrades non déformées. D pouvant en effet atteindre 42,5 $\mu$ lorsqu'il est mesuré sur des tétrades totalement déformées.

Annales de l'Abeille. - I966. 
II. Daboecia polifolia DoN. (Andromeda dabaecu L. Menziezia polifolia Juss. Menziezia dabaecu D. C. Vaccinium cantabricum HUDS. Boretta cantabrica O. KUNTZE. Boretta dabaecu BAILLON.

"Daboecie à feuilles de Polium ».

Forme et symétrie : pollen en tétrade assez peu globuleuse rappelant celle d'Erica vagans, les pôles des grains cependant sont moins nettement aplatis que chez cette dernière.

Dimensions en $\mu$ :

$$
\begin{array}{ll}
\mathrm{D}=32 & (29,2<\mathrm{D}<33,5) \\
d=2 \mathrm{I} & (\mathrm{I} 9,8<d<23) \\
g=2 \mathrm{I}, 5 & (\mathrm{I} 9,5<g<23,2) \\
p=5,7 & (4,6<p<6,5)
\end{array}
$$

Apertures : 6 paires de demi-sillons.

Sillons étroits à limites peu nettes, assez effilés atteignant Ig $\mu$ de long.

Pores : petits, circulaires, très souvent absents.

Marges : peu importantes à inexistantes.

Exine : ensemble ectexine-endexine ne dépassant pas $I, 6 \mu$, ectexine bacculée, à éléments fins et très serrés.

Intine : plus mince que 1'exine, claire.

Cytoplasme : clair, irrégulièrement granuleux.

12. Loiseleuria procumbens DESV. (Azalea procumbens L.)

«I,oiseleurie couchée ».

Forme et symétrie: pollen en tétrade calymmée, très régulière, fortement lobée.

Dimensions (en $\mu$ ) :

$$
\begin{array}{ll}
\mathrm{D}=29,5 & (27,6<\mathrm{D}<3 \mathrm{I}, 2) \\
d=20 & (\mathrm{I} 8,5<d<22,3) \\
g=20,5 & (\mathrm{I} 8,5<g<22) \\
p=\mathrm{IO} & (9,2<p<\mathrm{I} 0, \mathrm{I})
\end{array}
$$

Apertures : 6 paires de demi-sillons.

Sillons courts médians ( $2 f$ ne dépassant pas I3 $\mu$, et $\varepsilon 2,2$ à $2,5 \mu$ ). Extrémités des sillons arrondies ou très légèrement pointues.

Pores : souvent absents, circulaires, à contours nets lorsqu'ils existent $2,5 \mu$ de diamètre environ.

Marges : très réduites, voire nulles.

Exine : endexine plus épaisse que l'ectexine, l'ensemble ne dépassant pas $I, 7 \mu$ d'épaisseur.

Intine : mince, épaisseur inférieure à celle de l'exine.

Cytoplasme : clair, très faiblement granuleux. 
13. Phyllodoce caerulea FRIES. (Andromeda caerulea L. Andromeda taxifolia PALL. Erica carulea WILD. Menziezia caerulea SM. Bryanthus taxifolius GRAY. Phyllodoce taxifolia saLisB.

"Phyllodoce bleue ".

Forme et symétrie : pollen en tétrade calymmée, de forme très souvent irrégulière, tendance comme chez Calluna vulgaris à la présentation des 4 grains dans un même plan, pôles des grains aplatis de façon caractéristique.

Dimensions (en $\mu$ ) :

$$
\begin{array}{ll}
\mathrm{D}=32,5 & (30<\mathrm{D}<34,5) \\
d=23 & (2 \mathrm{I}<d<25,6) \\
g=23,5 & (2 \mathrm{I}<g<25,7) \\
p=9,2 & (6,5<p<\mathrm{I} 3, \mathrm{I})
\end{array}
$$

Apertures : 6 paires de demi-sillons.

Sillons allongés, étroits, à bords peu nets recouverts par les granules de l'ectexine. ( $2 f=20 \mu$ environ).

Pores : légèrement ellipsoïdes, assez étroits, font fréquemment défaut.

Marges : épaississements de l'endexine peu importants mais s'étendant latéralement sur plus de $3 \mu$.

Exine : endexine plus épaisse que l'ectexine, l'ensemble atteignant $2 \mu$. Éléments de sculpture très petits et irrégulièrement disposés : exine "scabre ".

Intine : épaisse, épaisseur atteignant celle de l'exine.

Cytoplasme : clair et granuleux.

\section{I4. Andromeda polifolia L.}

" Andromédie à feuilles de Polium ".

Forme et symétrie : pollen en títrade plus ou moins régulière, aspect "fripé " fréquent, pôles des grains irrégulièrement aplatis : ensemble de caractères qui donne au pollen une allure très polymorphe.

Dimensions (en $\mu$ ) :

$$
\begin{array}{ll}
\mathrm{D}=42 & (40,2<\mathrm{D}<44) \\
d=3 \mathrm{I}, 3 & (29,4<d<35) \\
g=33,5 & (3 \mathrm{I}, 3<g<36) \\
p=\mathrm{I} 6 & (\mathrm{I} 3<p<\mathrm{I} 8,5)
\end{array}
$$

Apertures : 6 paires de demi-sillons.

Sillons courts (I $8 \mu<2 f<23 \mu$ ) à extrémités pointues mais peu effilées.

Pores : circulaires, diamètre atteignant $4,5 \mu$. Anneaux autour des pores particulièrement visibles.

Marges : bien marquées et à extension latérale importante : 4,5 $\mu$.

Exine : épaisse : ectexine difficilement discernable de l'endexine, l'ensemble atteignant 3,2 $\mu$ d'épaisseur. Exine verrulée, éléments de sculpture très rapprochés par endroits tendant à constituer un réticulum. Ornementation plus distincte dans les intercolpiums qu'aux pôles. 
Intine : très mince, (I,2 $\mu$ d'épaisseur), claire.

Cytoplasme : granuleux.

I5. Erica arborea L. (Erica procera SALISB. Erica elata BUBANI.)

"Bruyère arborescente".

Forme et symétrie : pollen en tétrade calymmée, tétrade bien lobée, pôles des grains légèrement aplatis, murs internes de la tétrade très épais $(2,7 \mu)$, caractéristiques du genre.

Dimensions (en $\mu$ ):

$$
\begin{array}{ll}
\mathrm{I})=32,2 & (3 \mathrm{I}<\mathrm{D}<37,5) \\
d=83,5 & (20,4<d<26,7) \\
g=23 & (2 \mathrm{I}, 5<g<26) \\
p=7,2 & (6,2<p<8, \mathrm{I})
\end{array}
$$

Apertures : 6 paires de demi-sillons.

Sillons assez longs $(2 f=20,6 \mu)$, assez étroits $\varepsilon=2,6 \mu$ environ, extrémités des sillons variables : souvent légèrement fourchues, parfois non fourchues et pointues, effilées à émoussées.

Pores et marges très difficilement visibles. Non mesurés.

Exine : épaisse : ensemble ectexine-endexine atteignant $3 \mu$ d'épaisseur, endexine plus épaisse que l'ectexine. Exine verrulée mais éléments de sculpture souvent très rapprochés tendant à constituer un pseudo-réticulum.

Intine : claire, beatcoup plus mince que l'exine.

Cytoplasme : granuleux.

I6. Erica scoparia $\mathrm{L}$.

"Bruyère à balais ".

Forme et symétrie : pollen en tétrade calymmée, assez fortement lobée, muts internes de la tétrade épais.

Dimensions (en $\mu$ ) :

$$
\begin{array}{ll}
\mathrm{D}=33,2 & (30<\mathrm{D}<35,5) \\
d=24,7 & (23<d<25,4) \\
g=25 & (23,5<g<26) \\
p=7 & (6<p<8,2)
\end{array}
$$

Apertures : 6 paires de demi-sillons.

Sillons allongés $(2 f=2 \mathrm{I} \mu)$ assez larges $(\varepsilon=4,3 \mu)$. Bords des sillons rendus peu nets par la présence de granules d'ectexine, extrémités des sillons effilées parfois bifides.

Pores circulaires, parfois légèrement allongés, diamètre de 4,3 à 4,5 $\mu$ environ. Marges assez peu épaisses mais étendues ( 3 à $3,5 \mu$ d'extension latérale).

Exine : épaisse, endexine plus épaisse que l'ectexine; l'ensemble atteignant 2,3

à $3.5 \mu$ d'épaisseur. Exine réticulée, mailles du réticulum petites et régulières.

Intine : claire, plus mince que l'ensemble endexine-ectexine.

Cytoplasme : granuleux. 
17. Erica carnea $\mathrm{I}_{1}$. (E. saxatilis SALISB. E. herbacea BENTHAM.).

"Bruyère couleur de chair ".

Forme et symétrie : pollen en tétrade calymmée sub-sphérique, pôles des grains pas ou peu aplatis, murs internes de la tétrade minces.

Dimensions (en $\mu)$ :

$$
\begin{array}{ll}
\mathrm{D}=31,6 & (30<\mathrm{D}<33) \\
d=25,8 & (23,7<d<27,2) \\
g=26 & (24,3<g<27,3) \\
p=6 & (4,8<p<7,2)
\end{array}
$$

Apertures : 6 paires de demi-sillons.

Sillons particulièrement visibles, extrémités non effilées, souvent bifides, assez larges ( $\varepsilon$ atteignant $3,2 \mu$ ), longueur d'environ I $8 \mu$.

Pores : étroits et allongés rarement visibles.

Marges : peu importantes.

Exine : l'ensemble ectexine-endexine atteignant I, $8 \mu$ d'épaisseur ; ectexine à peu près aussi épaisse que l'endexine; exine verrulée, ornementation plus visible aux pôles des grains qu'à l'équateur.

Intine : claire, presque aussi épaisse que l'exine.

Cytoplasme : clair, parfois très peu granuleux.

I8. Erica tetralix L. (Erica botuliformis SALISB. Tetralix septentrionalis E. MAY.). "Bruyère à 4 angles ".

Forme et symétrie : pollen en tétrade calymmée régulière, pôles des grains aplatis. Dimensions $(e n \mu)$ :

$$
\begin{array}{ll}
\mathrm{D}=37,2 & (35,5<\mathrm{D}<38,3) \\
d=27,6 & (26,8<d<29,8) \\
g=27 & (26,2<g<29) \\
p=8,2 & (7,6<p<8,8)
\end{array}
$$

Apertures : 6 paires de demi-sillons.

Sillons courts et larges ( $\varepsilon$ atteignant $4,6 \mu$ ) bords des sillons nettement délimités. Marges : épaisses : épaisseur de $2,8 \mu$ environ, extension latérale des marges $=4 \mu$.

Pores : clairs légèrement ellipsoïdes.

Exine : endexine plus épaisse que l'ectexine, l'ensemble atteignant 2,2 $\mu$ d'épaisseur. Exine verrulée, ornementation diminuant en importance lorsqu'on s'approche des pôles.

Intine : claire et mince, moins épaisse que l'ensemble endexine-ectexine. Cytoplasme : fortement granuleux (rappelant celui d'Erica cinera.) 
I9. Erica vagans L. (Erica decipiens ST-AMAND Erica didyma sTokEs Gypsocallis vagans GRAY.).

"Bruyère vagabonde ".

Forme et symétrie : pollen en tétrade calymmée, sub-triangulaire, tétrade bien lobée, pôles des grains très aplatis.

Dimensions $(e n \mu)$ :

$$
\begin{array}{ll}
\mathrm{D}=29,2 & (27<\mathrm{D}<3 \mathrm{I}, 8) \\
d=20 & (\mathrm{I} 8<d<2 \mathrm{I}, 7) \\
g=20,5 & (\mathrm{I} 8<g<2 \mathrm{I}, 5) \\
p=6,8 & (5<p<8,2)
\end{array}
$$

Apertures : 6 paires de demi-sillons.

Sillons longs $(2 f=I 8 \mu)$ et étroits (largeur des sillons à l'équateur ne dépassant pas $I, 5 \mu$ ) bords des sillons parfois irréguliers, extrémités des sillons très effilées, parfois nettement bifides.

Pores : petits, clairs, allongés souvent absents.

Marges : claires, assez épaisses, peuvent s'étendre latéralement sur 3 à $3,5 \mu$.

Exine : assez fine, ensemble ectexine-endexine ne dépassant pas 2 à $2,2 \mu$; endexine plus épaisse que l'ectexine, exine verrulée, ornementation constituée de très petites protubérances.

Intine : claire, plus mince que l'ensemble ectexine-endexine.

Cytoplasme : très clair, non granuleux.

20. Erica cinera L. (Erica tenuifolia BUBANI. Erica viridi purpureum GOUAN non I. Erica mutabilis SALISB. Erica humilis NECKER.).

(Bruyère cendrée ».

Espèce remarquable par l'ensemble de ses caractères.

Symétrie et forme : pollen en tétrade calymmée, sub-sphérique, pôles des grains souvent aplatis.

Dimensions (en $\mu$ ) :

$$
\begin{array}{ll}
\mathrm{D}=4^{2} & (40<\mathrm{D}<43,6) \\
d=3^{2} & (30,7<d<33,6) \\
g=29 & (27<g<3 \mathrm{I}) \\
p=\mathrm{I} 2,2 & (\mathrm{II}, 2<p<\mathrm{I} 3)
\end{array}
$$

Apertures : 6 paires de demi-sillons.

Sillons longs et larges $2 f=24$ à $25 \mu, \varepsilon$ dépassant $4 \mu$, bords des sillons irréguliers, extrémités bifides des sillons caractéristiques du genre.

Pores : très visibles, sub-circulaires pouvant dépasser $4 \mu$ de diamètre, anneaux autour des pores peu marqués.

Marges : extension latérale faible mais épaississements très marqués de 1'endexine $(2,3 \mu$ d'épaisseur $)$. 
Exine : endexine plus épaisse que l'ectexine, l'ensemble atteignant $2,2 \mu$ d'épaisseur

Exine finement et régulièrement réticulée, mailles du réticulum plus petites aux pôles qu'à l'équateur de chaque grain.

Intine : plus claire que l'exine et presque aussi épaisse.

Cytoplasme : très granuleux, caractéristique.

2I. Erica multiflora L. (Gypsocallis multiflora Don. Erica umbellifera $\left.\mathrm{L}_{\mathrm{O}} \mathrm{OrS}.\right)$. "Bruyère à fleurs nombreuses ".

Symétrie et forme : pollen en tétrade fortement lobée, pôles des grains très aplatis.

Dimensions (en $\mu$ ) :

$$
\begin{array}{ll}
\mathrm{D}=3 \mathrm{I}, 6 & (29,2<\mathrm{D}<33) \\
\vec{d}=\mathrm{I} 9,2 & (\mathrm{I} 8,3<d<20,5) \\
g=\mathrm{I} 9 & (\mathrm{I} 8<g<20,5) \\
p=6,2 & (5<p<7,2)
\end{array}
$$

Apertures : 6 paires de demi-sillons.

Sillons allongés $(2 f$ atteignant $2 \mathrm{I} \mu$ ) et assez étroits $(\varepsilon=\mathrm{I}, 8$ à $2 \mu)$.

Pores : elliptiques, difficilement visibles, peuvent faire défaut.

Marges : très peu importantes à inexistantes.

Exine : endexine plus épaisse que l'ectexine, l'ensemble atteignant $2,6 \mu$ d'épaisseur.

Exine verrulée, disposition des éléments de sculpture irrégulière.

Intine : Claire, beaucoup plus mince que l'ensemble endexine-ectexine.

Cytoplasme : clair, parfois finement granuleux. LINDB.)

22. Erica lusitanica RUD. (Erica polytrichifolia SALISB.) (Erica codonodes

Forme et symétrie : pollen en tétrade calymmée, tétrade très souvent irrégulière ayant fréquemment un aspect froissé. Grains aplatis aux pôles.

Dimensions (en $\mu$ ) :

$$
\begin{array}{ll}
\mathrm{D}=34,2 & (32,3<\mathrm{D}<35,5) \\
d=23,6 & (22<d<24,2) \\
g=23,5 & (22<g<24,5) \\
p=\text { II }, 9 & (\text { IO }<p<12,8)
\end{array}
$$

Apertures : 6 paires de demi-sillons.

Sillons étroits, allongés ( $2 f$ ne dépassant pas I5 $\mu, \varepsilon=\mathrm{I}$ à $\mathrm{I}, 3 \mu$ ).

Pores : sub-circulaires, $2 \mu$ de diamètre environ, pas toujours visibles. Anneaux très nets autour des pores.

Marges : assez épaisses pouvant s'étendre latéralement sur 2 à $2,6 \mu$.

Exine : ectexine et endexine d'égale épaisseur, l'ensemble atteignant 2 à $2,2 \mu$ d'épaisseur.

Intine : claire, légèrement moins épaisse que l'exine.

Cytoplasme : clair, peu granuleux dans certains grains, plus granuleux dans d'autres. 
23. Erica ciliaris I. (Erica watsoni D.C. E. glandulosa CHEV.). "Bruyère ciliée ".

Forme et symétrie : pollen en tétrade calymmée sub-sphérique; pôles des grains

légèrement aplatis, tétrade peu lobée.

Dimensions $(\mathrm{en} \mu)$ :

$$
\begin{array}{ll}
\mathrm{D}=37,4 & (35,2<\mathrm{D}<39) \\
d=28 & (26,8<d<29,3) \\
g=28,3 & (26,5<g<30) \\
p=5,2 & (4<p<6)
\end{array}
$$

Apertures : 6 paires de demi-sillons.

Sillons longs $(2 f=25 \mu)$ se terminant donc près des pôles de chaque grain, et peu larges $(\varepsilon=3$ à $3,5 \mu)$, extrémités des sillons effilées, parfois légèrement ramifiées.

Pores : circulaires : diamètres atteignant 4,5 à $5 \mu$, parfois éloignés du lieu de jonction de 2 grains, peuvent faire défaut.

Marges : presque inexistantes.

Exine : ectexine moins épaisse que l'endexine. L'ensemble est peu épais, ne dépassant pas $2 \mu$ d'épaisseur. Exine réticulée, réticulum devenant de moins en moins visible au fur et à mesure qu'on s'approche des pôles.

Intine : claire, moins épaisse que l'ensemble endexine-ectexine.

Cytoplasme : très clair, pas ou très peu granuleux.

24. Erica mediterranea L. (Erica carnea variété occidentalis BEN'TH. Erica hybernica syme. Erica lugubris SALISB.).

"Bruyère de la méditerranée ".

Forme et symétrie : pollen en tétrade calymmée, de formeirrégulière : sub-sphérique et globuleuse à forme sub-triangulaire et peu globuleuse. Pôles des grains non aplatis, murs internes de la tétrade minces.

Dimensions en $\mu$

$$
\begin{array}{ll}
\mathrm{D}=30 & (28,7<\mathrm{D}<3 \mathrm{I}, 2) \\
d=22,5 & (2 \mathrm{I}, 6<d<23,5) \\
g=22 & (2 \mathrm{I}<g<23,4) \\
p=6 & (4,6<p<7,2)
\end{array}
$$

Apertures : 6 paires de demi-sillons.

Sillons longs $(2 f=I 8 \mu$ ) et étroits à terminaison très effilée caractéristique, dans quelques cas terminaisons légèrement bifides.

Pores : très petits ne dépassant pas $2 \mu$ de diamètre, font souvent défaut.

Marges : épaississements nets de l'endexine (2 à $2,3 \mu$ ), extension latérale très faible.

Exine : endexine plus épaisse que l'ectexine, l'ensemble atteignant $2,3 \mu$ d'épaisseur. Éléments de sculpture de l'exine s'amicinssant à leur base : exine gemmulée.

Intine : claire, plus mince que l'ensemble endexine-ectexine.

Cytoplasme : clair, peu granuleux. 
25. Erica umbellata GRAY.

Forme et symétrie : pollen en tétrade très lobée, grains fortement individualisés, donc en contact par une faible proportion de leur surface, méat visible entre les grains : aspect caractéristique.

Dimensions en $\mu$ :

$$
\begin{array}{lll}
\mathrm{D}=39,3 & (37 & <\mathrm{D}<42,3) \\
d=25,2 & (23,7<d<27) \\
g=25,8 & (23<g<26,7) \\
p=\text { n'a pu être mesuré. } &
\end{array}
$$

Apertures : 6 paires de demi-sillons.

Sillons clairs et étroits atteignant $24 \mu$ de long, extrémités des sillons le plus souvent émoussées, bifides dans quelques cas.

Marges : épaisses : $2,3 \mu$ d'épaisseur à faible extension latérale.

Pores : non mesurés, elliptiques, rarement visibles.

Exine : ensemble ectexine-endexine atteignant 2 à 2,4 $\mu$ d'épaisseur. Exine verrulée

Eictexine ne paraissant pas continue d'un grain à l'autre : tétrade acalymmée.

Cytoplasme : très granuleux.

26. Erica stricta DON. (Erica terminalis WILD.)

Forme et symétrie : pollen remarquable par le fait qu'il ne s'agit pas ici de tétrade.

Grains de pollen isolés, tricolpés, sub-sphériques.

Dimensions en $\mu$ :

$$
\begin{array}{ll}
\mathrm{P}=\text { longueur de l'axe polaire }=27,5 & (24<\mathrm{P}<29,5) \\
\mathrm{E}=\text { diamètre équatorial }=25,6 & (22,3<\mathrm{E}<271 \\
p=\text { côté du triangle polaire }=8,5 & (6,5<p<\text { IO })
\end{array}
$$

Apertures : pollen tricolporé :

3 sillons allongés $(2 f=\mathrm{I} 9 \mu)$, largeur des sillons à 1'équateur : $2,5 \mu$ environ. Pores : 3 pores ( 1 par sillon) en position sub-équatoriale : ces pores sub-circulaires occupent une position qui tend vers celle des pores des autres espèces d'Éricacées possédant un pollen en tétrade.

Marges : endexine légèrement épaisse sur les bords du sillon, marges s'étendant latéralement sur $3 \mu$ environ.

Exine : ectexine fine, l'ensemble ectexine-endexine ne dépassant pas $2 \mu$ d'épaisseur

Éléments de sculpture de l'exine petits : exine faiblement verrulée. Deux

couches sont discernables dans l'endexine de certains grains.

Intine : claire, presque aussi épaisse que l'ensemble ectexine-endexine.

Cytoplasme : clair, finement granuleux.

\section{$4^{\circ}$ Conclusion}

Les caractères qui permettent de distinguer deux espèces sont nombreux, certains étant plus particulièrement dignes d'intérêt. La forme du pollen pour ce qui est des Éricacées n'est généralement pas un caractère spécifique, abstraction faite 
de quelques cas particuliers (Calluna vulgaris, Erica umbellata, E. terminalis, par exemple). L'ornementation, par contre, constitue un bon critère; les dimensions, quoique susceptibles de fortes variations au moins pour certaines espèces, semblent constituer une bonne référence si elles sont évaluées statistiquement.

L'étude des apertures — et plus précisément des dimensions et des extrémités des sillons - est également d'un bon secours pour la détermination du pollen des Ericacées. Cette détermination, bien que souvent délicate, est donc possible si l'on s'appuie sur un ensemble de caractères.

La distinction a pu être faite pour les 26 espèces d'Éricacées étudiées, mais notons qu'elle est plus particulièrement délicate dans le cas des différentes espèces du genre Vaccinium, ainsi que dans celui des deux espèces de Rhododendron.

Par ailleurs, la différenciation Vaccinium myrtillus-Erica ciliaris n'est pas toujours très aisée non plus.

Nous avons tenté, en essayant de ne retenir que les caractères les plus décisifs, d'élaborer une clé de détermination du pollen "frais" des Éricacées.

\section{Clé de détermination du pollen frais des Éricacées}

Grains de pollen subsphériques tricolporés non réunis en tétrade, $p>6 \mu$ cytoplasme clair, finement granuleux Erica stricta DON.

Grains de pollen réunis en tétrade:

- méat visible au centre de la tétrade, grains en contact par une faible proportion de leur surface, grains bien individualisés Erica umbelleta GRAY;

- pas de méat visible au centre de la tétrade: grains en contact par une forte proportion de leur surface:

Ectexine s'épaississant aux pôles de chaque grain, parois internes de la tétrade épaisses.
Sillon large, pointu, cytoplasme clair $\mathrm{J}=38 \mu$ :
1
Rhododendron ferrugineum $\mathrm{L}$.
$\mathrm{D}>34 \mu \cdot \begin{aligned} & \text { Cytoplasme plus granuleux, } \mathrm{D}=40 \mu, \text { murs internes de } \\ & \text { la tétrade plus réguliers, plus rectilignes que dans le cas } \\ & \text { précédent: }\end{aligned}$

Éctexine pas ou peu épaisse aux pôles de chaque grain.

Tétrade sub-sphérique compacte $\mathrm{D}>3^{8} \mu$, cytoplasme toujours granuleux

$D>50 \mu$ exine très légèrement granuleuse:

Arbutus unedo. $\mathrm{I}$.

$$
\mathrm{D}<50 \mu\left\{\begin{array}{r}
44 \mu<\mathrm{D}<50 \mu, \text { sillons longs pointus étroits exine mince, } \\
\text { marges peu importantes : } \\
\text { Arbutus alpina. I. }
\end{array}\right.
$$


Tétrade sub-globuleuse, légèrement lobée, murs internes de la tétrade très épais $(>3 \mu)$.

Sillons longs, pores circulaires à elliptiques très visibles, réticulum à mailles petites et régulières: Erica scoparia $\mathrm{L}$.

Sillons etfilés étroits, cytoplasme granuleux, pôles des grains aplatis, éléments de sculpture de l'exine très rapprochés, constituant un pseudo-réticulum : Evica arborea L.

Tétrade moins globuleuse, plus distinctement lobée $\mathrm{D}>34 \mu$ exine verrulée dont l'ornementation s'estompe au fur et à mesure qu'on s'approche des pôles, allure de la tétrade trés irrégulière : Andromeda polifolia $\mathrm{L}$.

$\mathrm{D}<34 \mu ; p>7 \mu$ extrémités des sillons arrondies ou légèrement pointues, pores rarement visibles, cytoplasme clair : Loiseleuria procumbens DEST.

$\mathrm{D}>30 \mu$ sillons très visibles à extrémités non effilées, parfois bifides, cytoplasme clair, marges peu importantes : Erica carnea L.

$\mathrm{D}>28 \mu$ marges nettes, pôles non aplatis, tétrade souvent sub-triangulaire, murs internes de la tétrade très peu épais ( $\mathrm{I}$ à $I, 5 \mu$ ).

$\mathrm{D}<3 \mathrm{I} \mu$ tétrade habituellement sub-triangulaire, terminaisons des sillons souvent bifides, marges bien visibles assez épaisses, à faible extension latérale : Erica mediterranea $\mathrm{I}_{6}$.

Pôles des grains peu aplatis, ornementation de l'exine très fine, généralement peu apparente.

$\mathrm{D}<33 \mu ; p<7 \mu$ exine bacculée à éléments très fins, cytoplasme clair parfois finement granuleux, pores petits : Daboecia polifolia

DON.

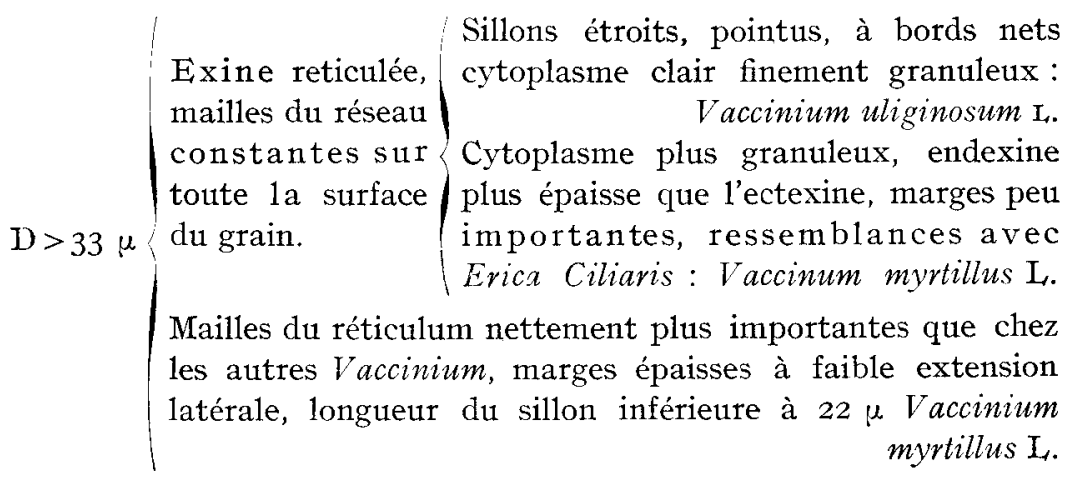

Tétrades plus fortement lobées, moins globuleuses, murs internes de la tétrade peu épais.

Épaisseur de l'exine supérieure à $2,3 \mu$, mailles du réticulum plus larges à l'équateur qu'aux pôles, pôles des grains peu aplatis : ectexine et endexine d'épaisseur sensiblement égales : Oxycoccos vulgaris 
Épaisseur de l'exine ne dépassant pas $2,2 \mu$, donc habituellement plus fine que chez les Oxycoccos, pôles des grains aplatis

Sillons courts, larges, nettement délinités, cytoplasme granuleux, marges importantes : Erica tetralix L.

Extrémités des sillons pointues parfois tifides, pores circu$d>27 \mu$ laires, endexine plus épaisse quel'ectexine : Erica ciliaris L. Sillons le plus souvent fourchus à l'extrémité, longueur $>20 \mu$, pores larges, cytoplasme très granuleux :

Erica cinerea L.

$d<26 \mu ; \not<9 \mu$ Tétrades sub-triangulaires, sillons allongés et étroits : Erica vagans L.

$d<26 \mu$ intine presque aussi épaisse que l'exine, pores petits subcirculaires, bordés par un anneau très net;

Erica lusitanica RUD.

Tétrade bien lobée, pôles des grains très aplatis, sillons pointus, intine peu épaisse, pores elliptiques :

Erica multifiora. I.

Tétrade très souvent irrégulière, jamais globuleuse, si la tétrade est régulière $d<30 \mu$ nette tendance à la présentation des 4 grains dans un même plan.

Sillons à bords peu nets et à extrémités difficilement visibles, pores de forme allongée, exine intectée : Phyllodoce cerulea FrIEs.

Cytoplasme très granuleux, sillons courts de forme irrégulière; pores peu visibles, exine tectée:

Calluna vulgaris SALISB.

La suite de notre travail nous a prouvé que parmi toutes les Ericaceae étudiées, seul un petit nombre d'espèces est important en matière d'analyse mellissologique, ce qui nous a amené à mettre au point la clé de détermination simplifiée que nous proposons ci-dessous :

pas de méat visible au centre de la tétrade, grains en contact par une forte proportion de leur surface :

ectexine s'épaississant aux pôles de chaque grain, tétrade sub-sphérique, $3 \mu<\mathrm{D}<40 \mu$, sillons pointus, parfois légèrement bifides assez courts $\left(2 f=\mathrm{I}_{5} \mu\right)$ : cytoplasme clair, homogène. Rhododendron ferrugineum $\mathrm{I}_{1}$. ectexine non épaissie aux pôles de chaque grain:

$D \simeq 50 \mu$, tétrade compacte. sillons allongés, larges, marges importantes, cytoplasme granuleux: Arbutus uned, I.

Tétrade sub-globuleuse $3 \mathrm{I} \mu<\mathrm{I})<33 \mu$ murs internes de la tétrade très épais, pćles peu aplatis, extrémités des sillons très variables (fourchues, émoussées, effilées) :

Erica arborea $\mathrm{L}$.

Tétrade plus distinctement lobée: murs internes de la tétrade non aussi épaisses.

Pôles non aplatis $30 \mu<D<33 \mu$ sillons très visibles allongés, extrémités non effilées, cytoplasme clair :

Erica carnea I. 
$\mathrm{D}>40 \mu$ sillons particulièrement fourchus aux extrémités, pores circulaires, cytoplasme très granuleux: Erica cinerea $\mathrm{L}$.

$35 \mu<\mathrm{D}<39 \mu\left\{\begin{array}{l}\text { Cytoplasme très clair, marges peu importantes, } \\ \text { sillons allongés étroits : } \\ \text { Cytoplasme plus granuleux, marges épaisses, } \\ \text { sillons courts et larges: }\end{array}\right.$

$27 \mu<\mathrm{D}<32 \mu$ tétrade subtriangulaire, pôles aplatis, sillons longs et étroits :

Erica vagans $\mathrm{L}$.

Tétrade très souvent irrégulière, avec tendance fréquente à la présentation des 4 grains dans un même plan, exine tectée, columelles bien visibles:

Calluna vulgaris LALISB.

Pôles des grains non aplatis $32 \mu<\mathrm{D}<38 \mu$ exine finement réticulée, marges peu importantes, cytoplasme peu granuleux :

Vaccinium myrtillus $\mathrm{L}$.

Méat visible au centre de la tétrade, grains bien individualisés en contact seulement par une faible proportion de leur surface :

Erica umbellata GRAY.

\section{II. - LES ÉRICACÉES DE FRANCE}

\section{A. - Distribution géographigue}

A l'exception de deux espèces, Erica umbellata et Erica stricta, toutes les Éricacées précédemment décrites se retrouvent en France; certaines ont une distribution géographique très large, d'autres ne sont présentes qu'en quelques localités (Phyllodoce cerulea, Erica lusitanica, Erica mediterranea, par exemples).

I1 nous a paru bon d'essayer de dresser des cartes de distribution géographique concernant les 26 espèces d'Éricacées précédemment citées. Ces cartes dont une partie figure dans cette publication (voir fig. 2 à I7), nous permettront de comparer ensuite les aires de distribution géographiqne et méllisologique. Les éléments nécessaires à la réalisation de ces cartes ont été empruntés à : G. BonNIER, I934; H. CostE, igo6 ; P. Dupont, P. I962 ; Fournier, I946; Hegi ; E. Hulten, I950 ; A.-F. JEANJEAN, I96I; M. Willkomm et J. LANGE, I870.

Ces cartes réalisées à l'échelle d'un pays n'ont évidemment de valeur que si elles sont considérées d'un point de vue général.

Elles permettent essentiellement de savoir si, dans une région donnée, telle espèce est fréquente ou pas, si son aire de distribution est de basse, moyenne ou haute altitude, si cette aire est fragmentaire ou globale, si la plante considérée est plus atlantique que méditerranéenne, plus septentrionale que méridionale, si c'est une plante de zone humide ou sèche, de régions à moyennes de températures basses ou élevées, préférant des sols plutôt calcaires qu'acides et réciproquement, ce qui nous amène à envisager au moins brièvement quelques points particuliers se rapportant à l'écologie des Éricacées. 


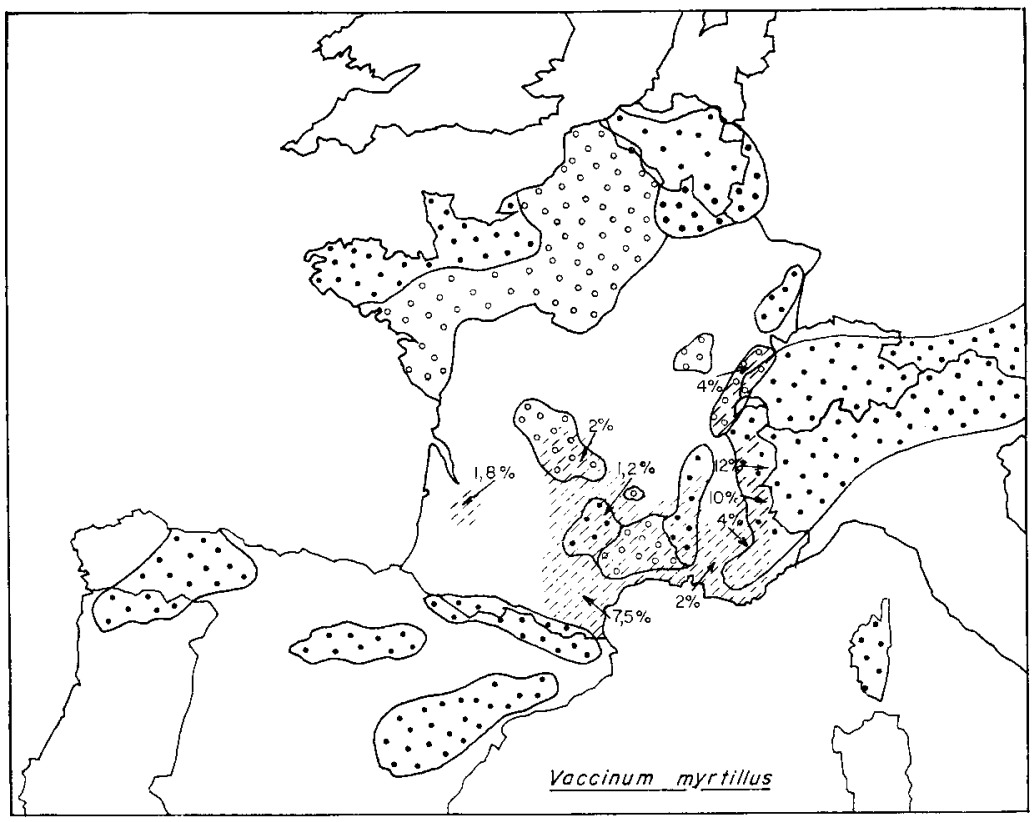

FIG. 2.

\section{Distribution géographique}

\section{$\because \because \because \because$ Fréquent à très fréquent}

: $: 0: 0$ fréquent à rare

Limites des aires géographiques telles qu'elles résultent de la bibliographie

2. Distribution melissologique (mode d'apparition_-du pollen)

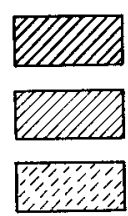

Dominant

Accompagnement

Isolé

Les pourcentages indiqués sur les cartes correspondent à la fréquence d'apparition dens les différentes régions. Dans certains cas on a tenté de tracer des courbes de niveau de la fréquence d'apparition. Les courbes n'ont qu'une valeur indicative et marquent des tendances générales non des valeurs absolues. 


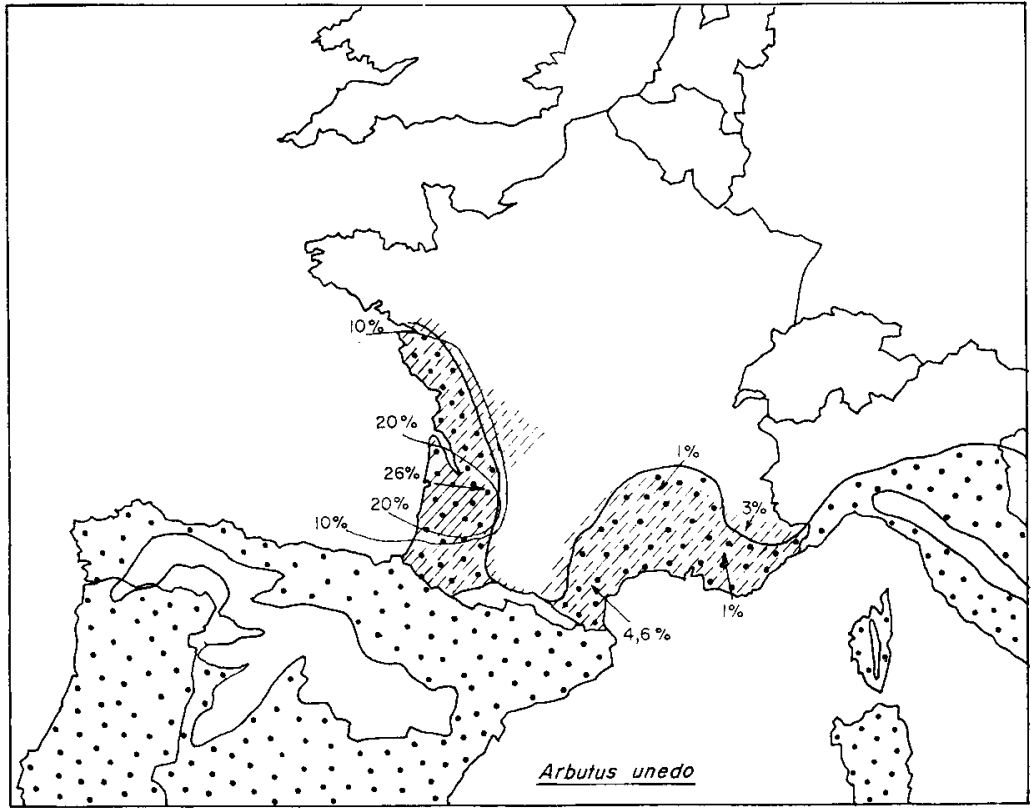

FIG. 3 .

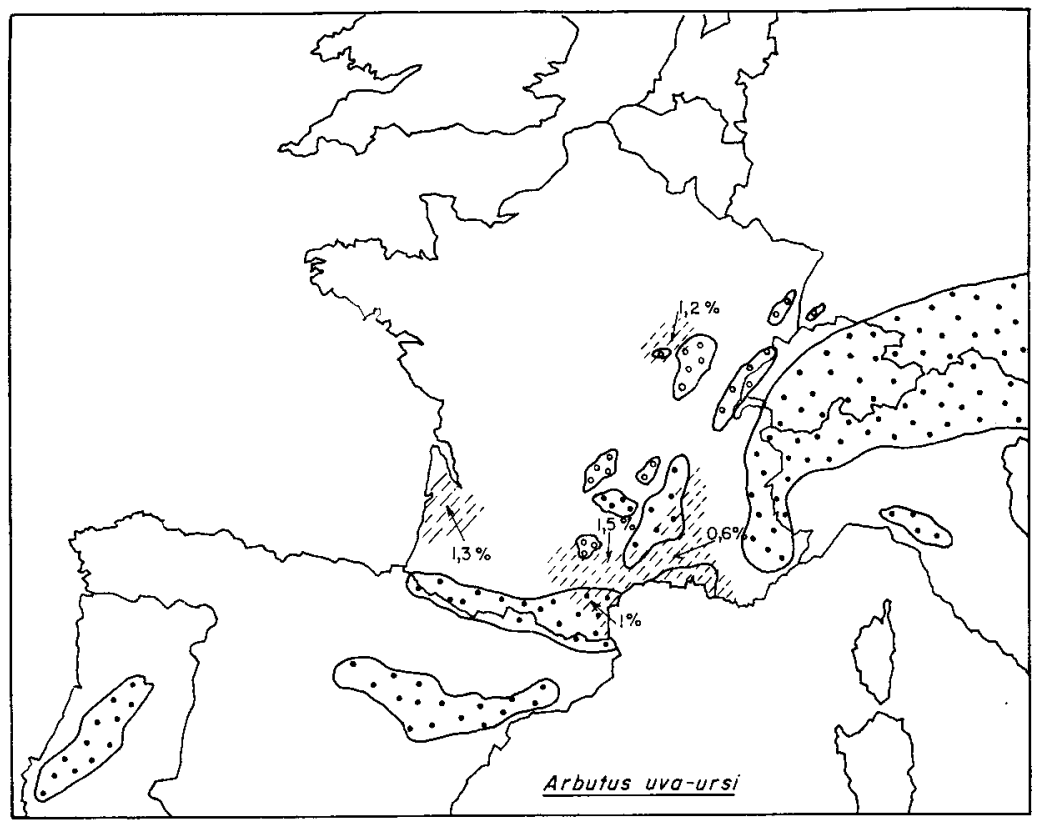

FIG. 4 . 


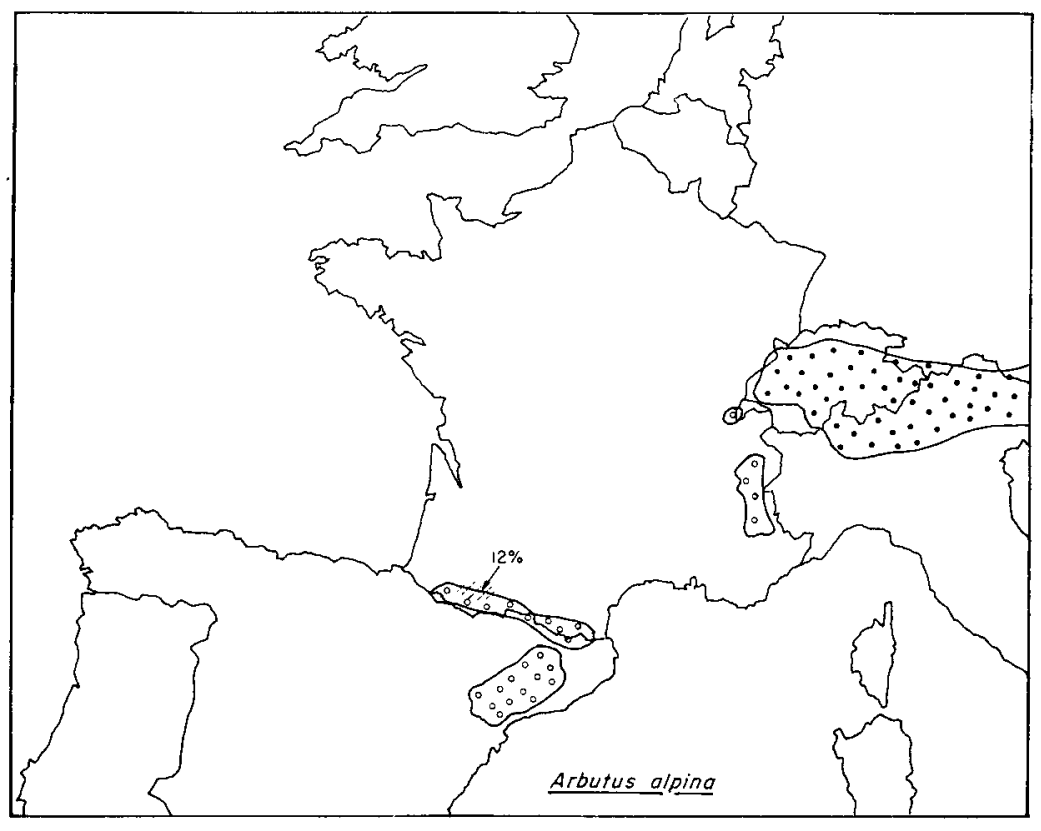

FIG. 5.

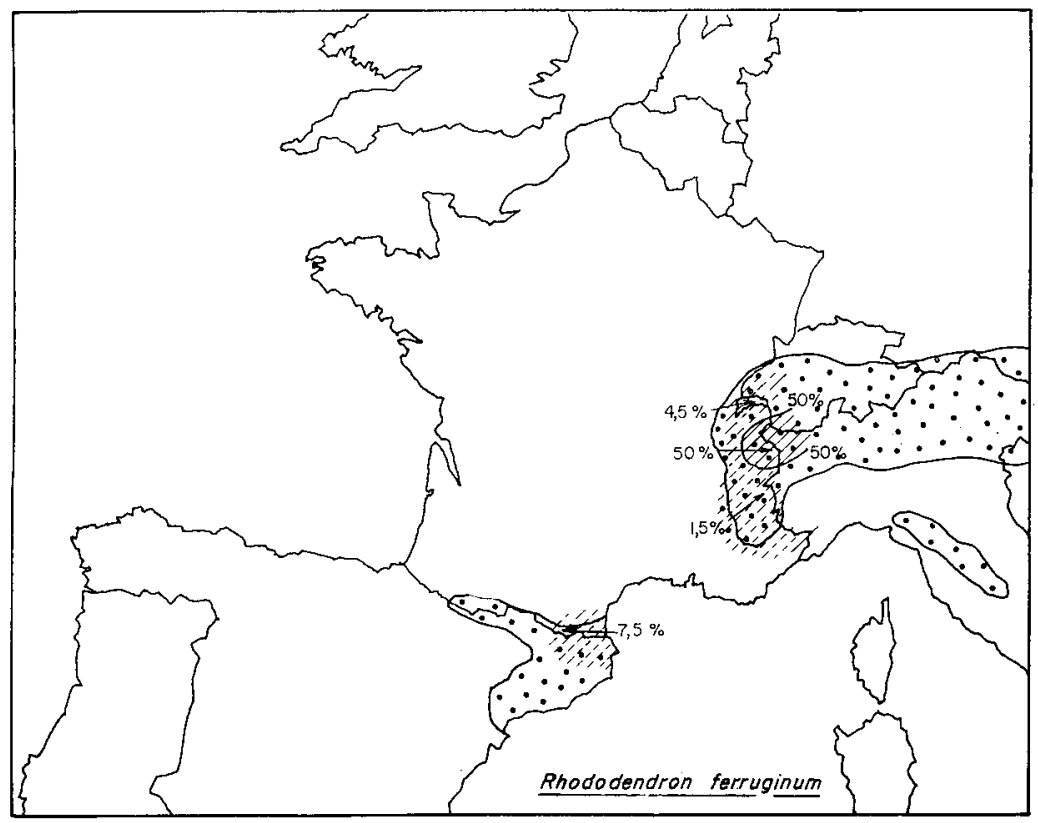

FIG. 6. 


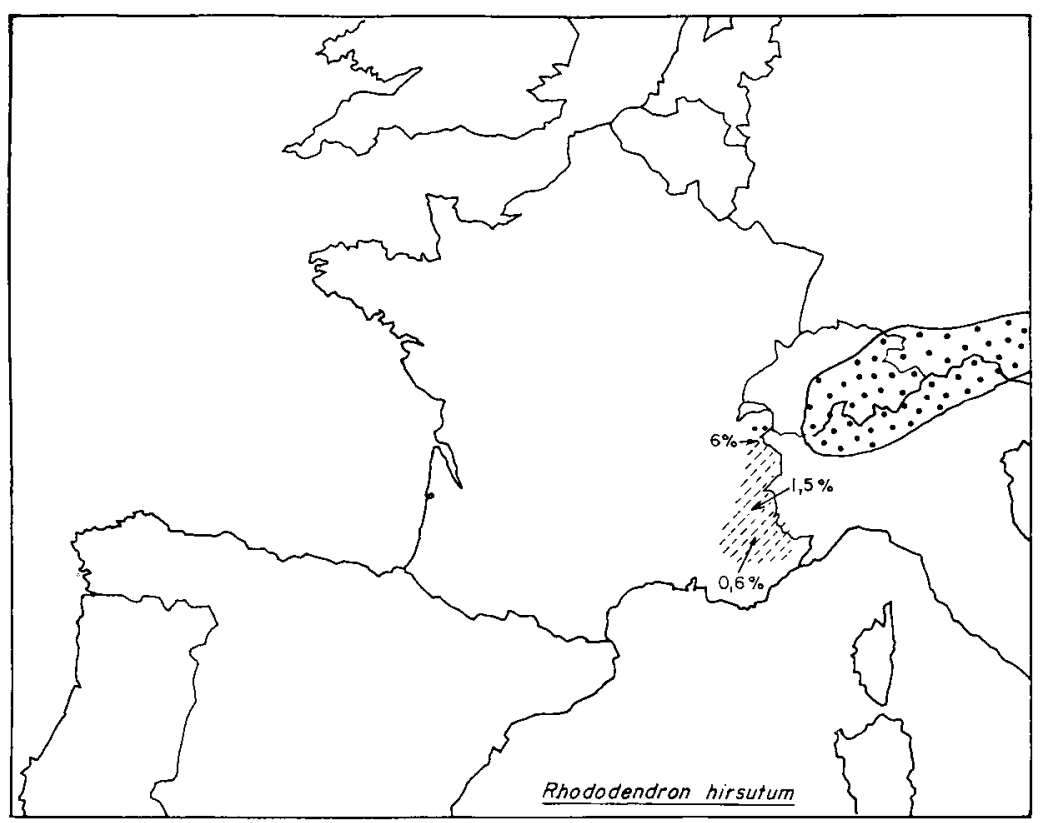

FIG. 7 .

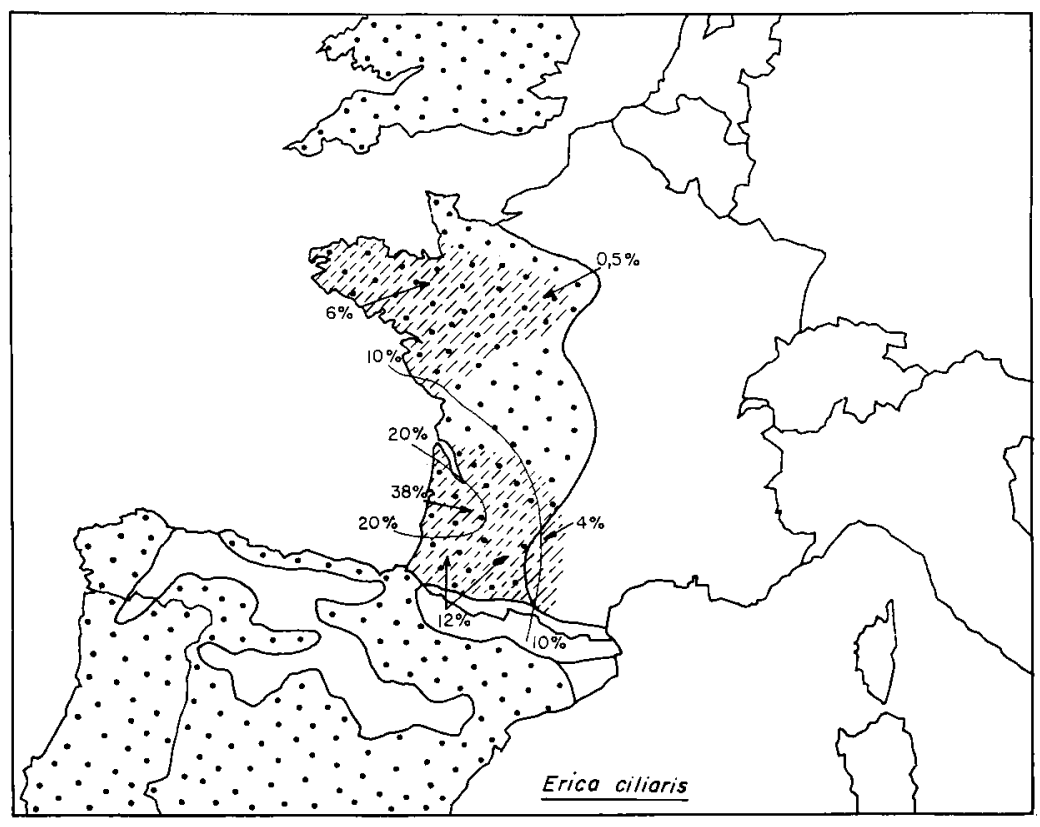

FIG. 8. 


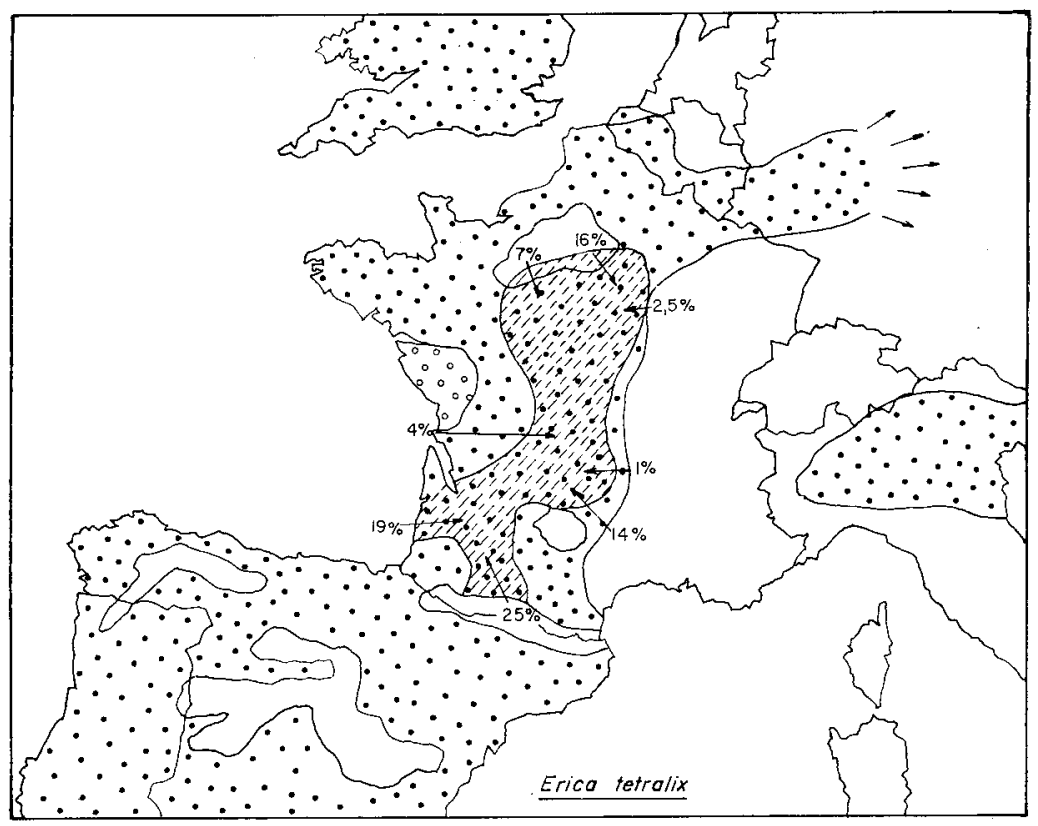

FIG. 9.

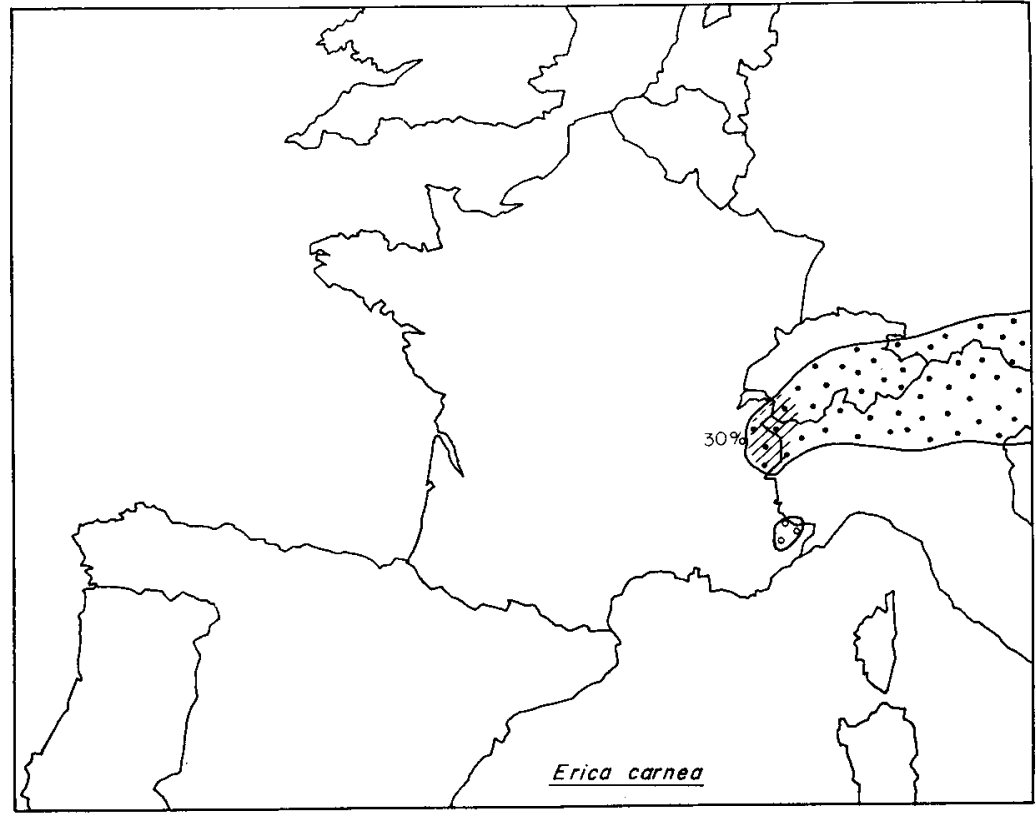

FIG. Io. 


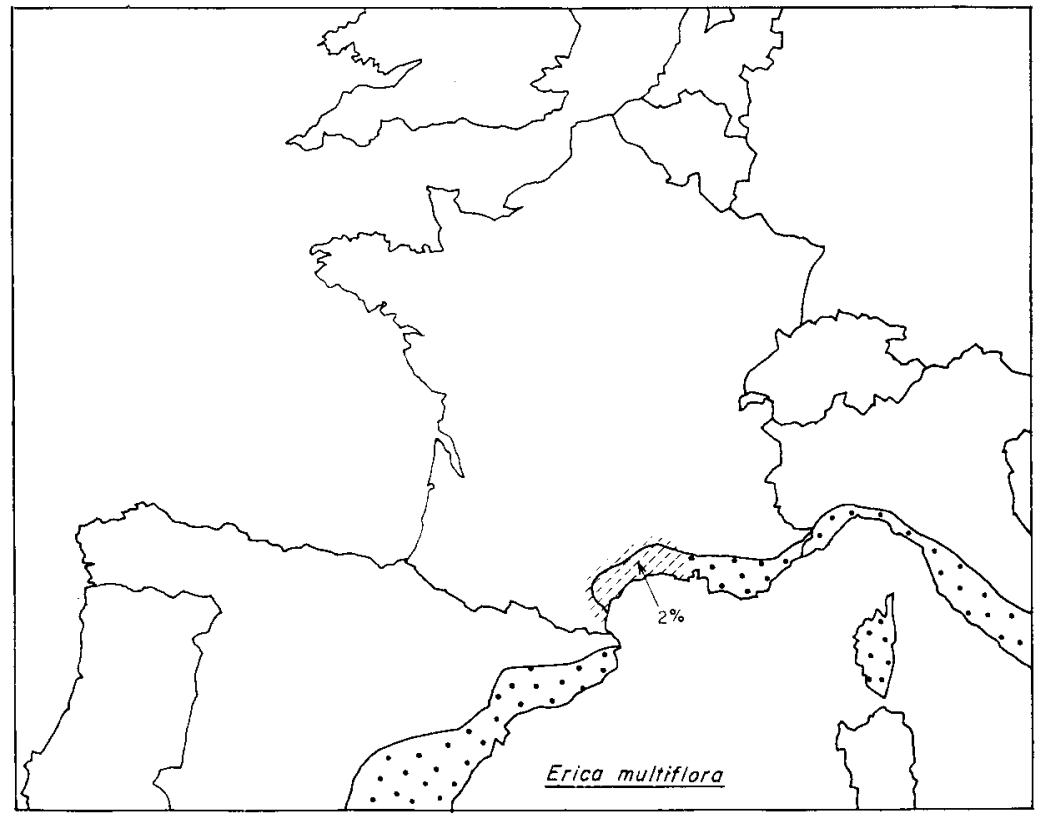

FIG. II.

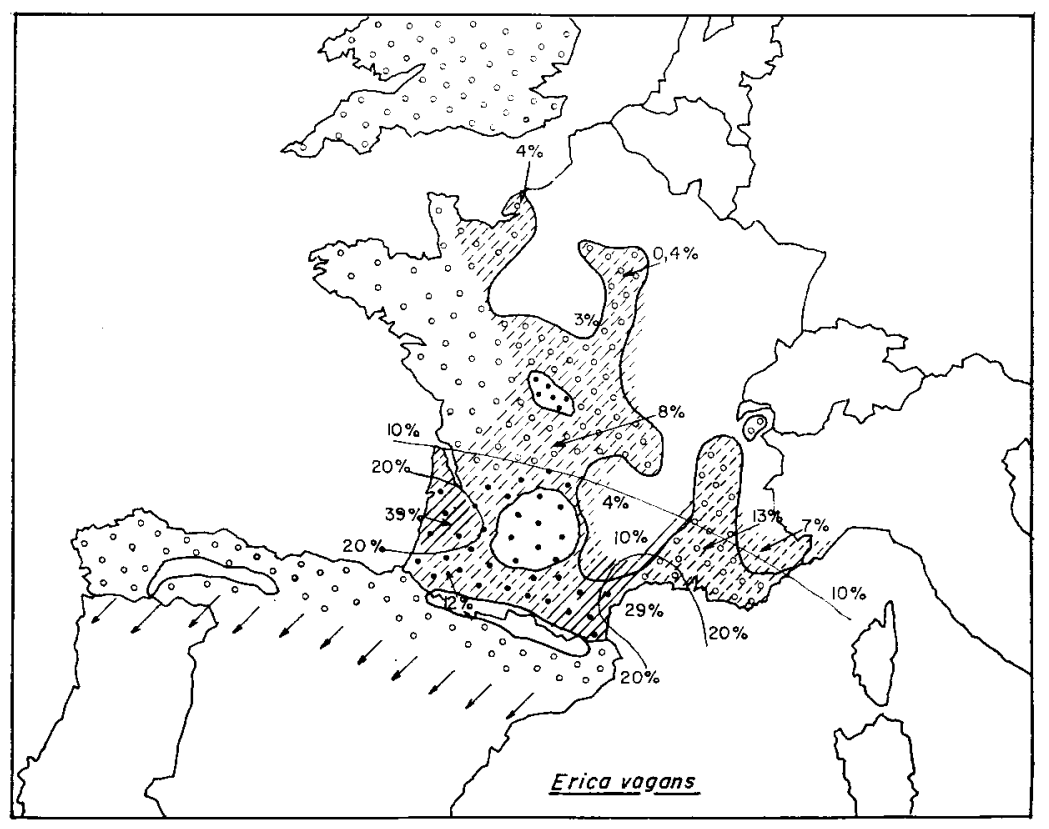

FIG. I2. 


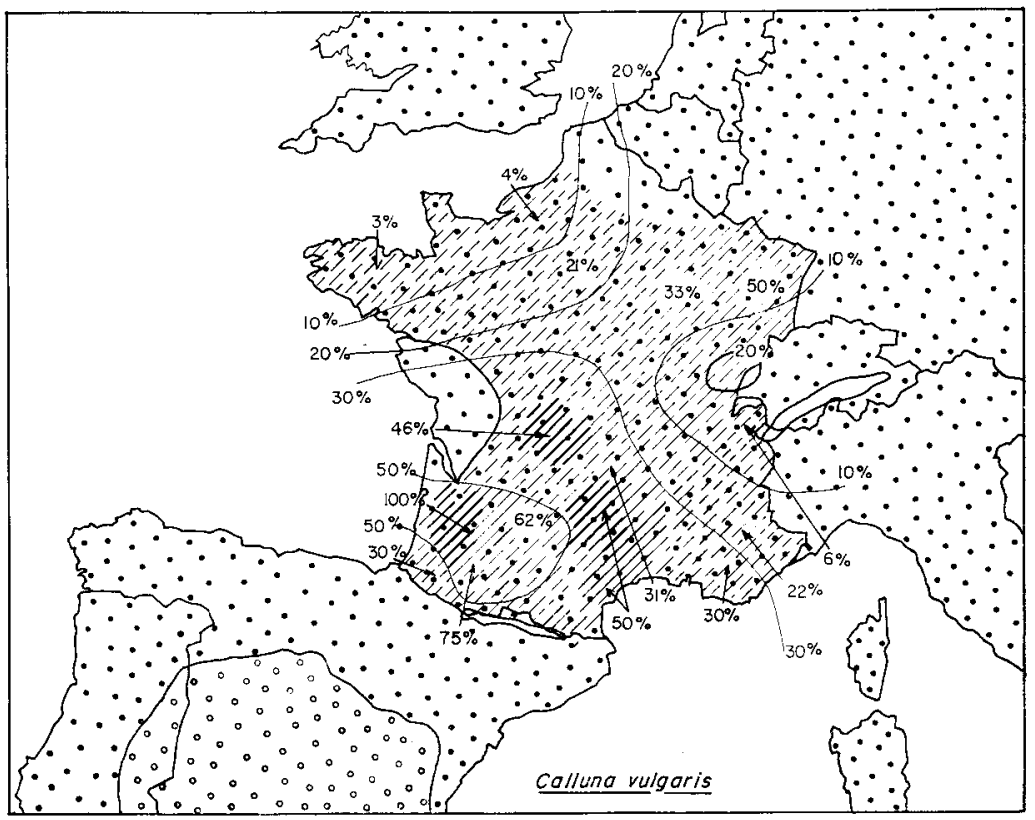

FIG. 13.

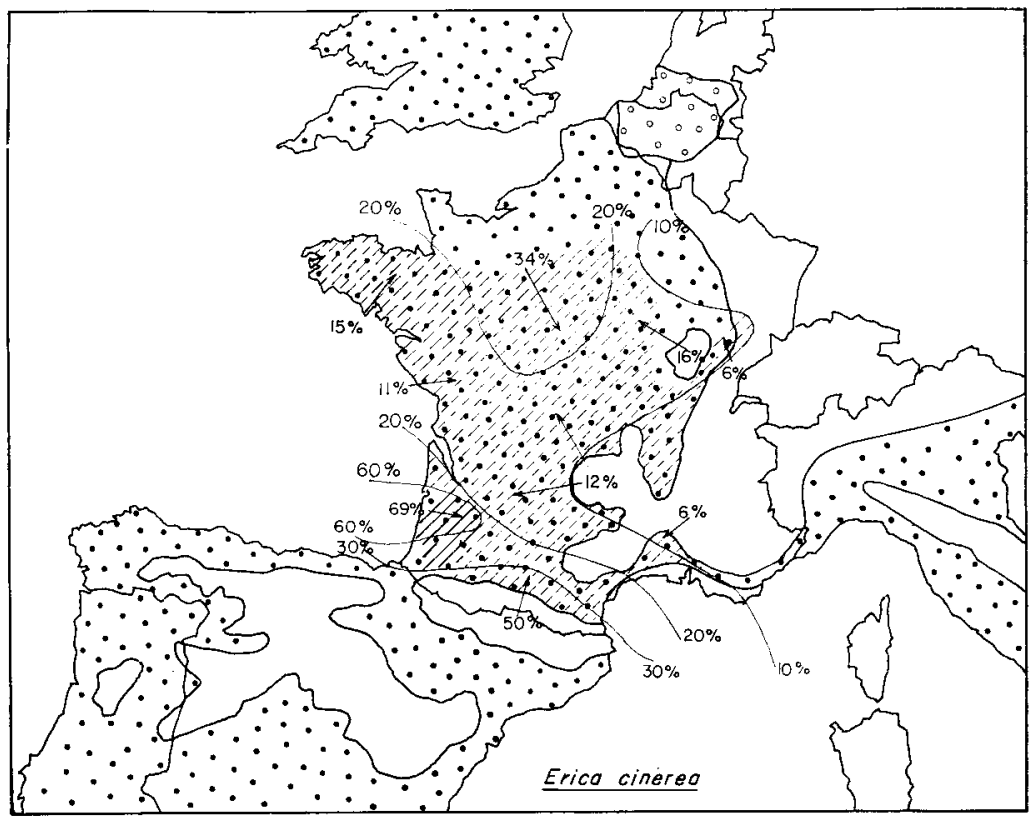

FIG. 14. 


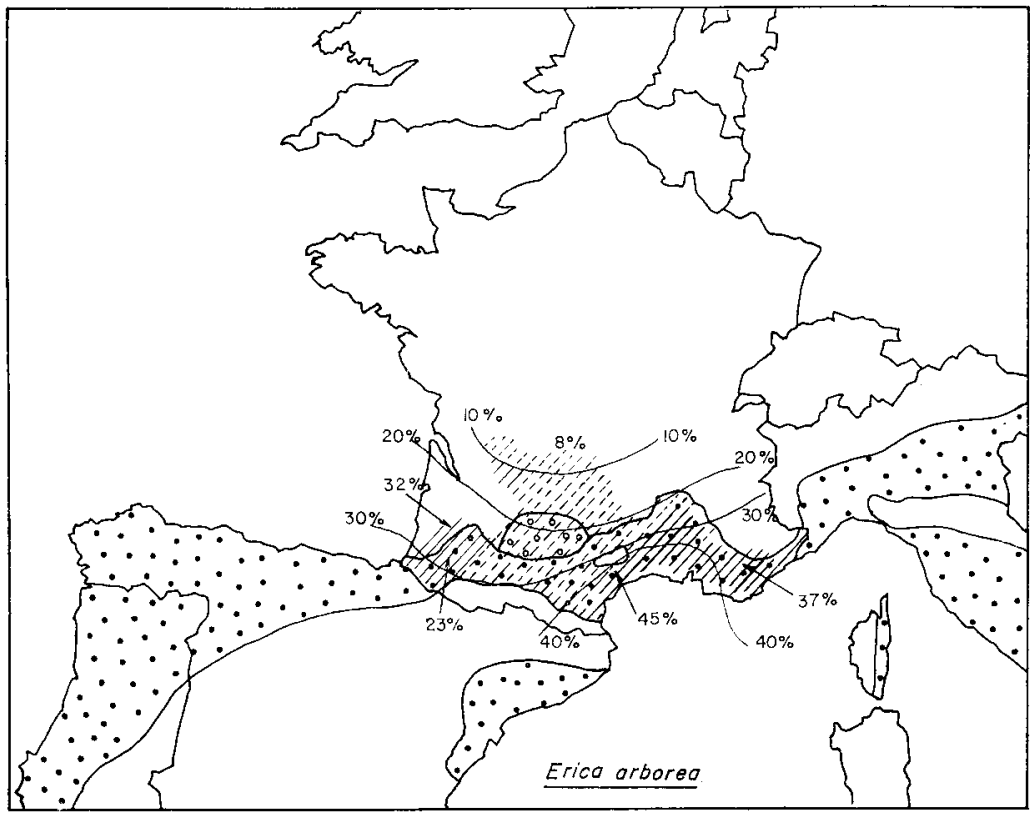

FIG. I5.

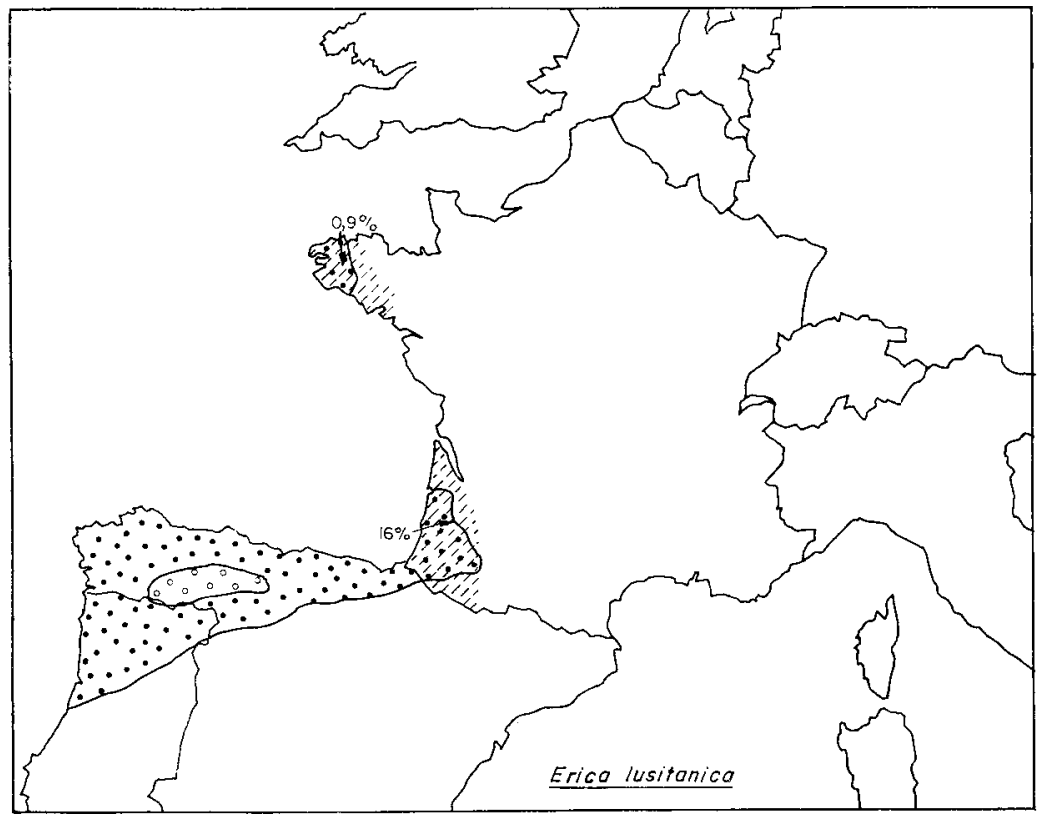

FIG. 16. 


\section{B. - ÉCOLOGIE DES ÉRICACÉES}

\section{Les Éricacées, plantes acidophiles}

Ce problème revêt, lorsqu'on s'adresse aux Éricacées, un caractère assez particulier. Peu de Végétatux en effet présentent autant d'aptitudes que les Éricacées à envahir les terrains maigres et épuisés J. (Grroux, I934). Ces caractères biologiques sont corroborés par la prédilection qu'affectent les Éricacées pour les terrains tourbeux dont la réaction très généralement acide est bien connue.

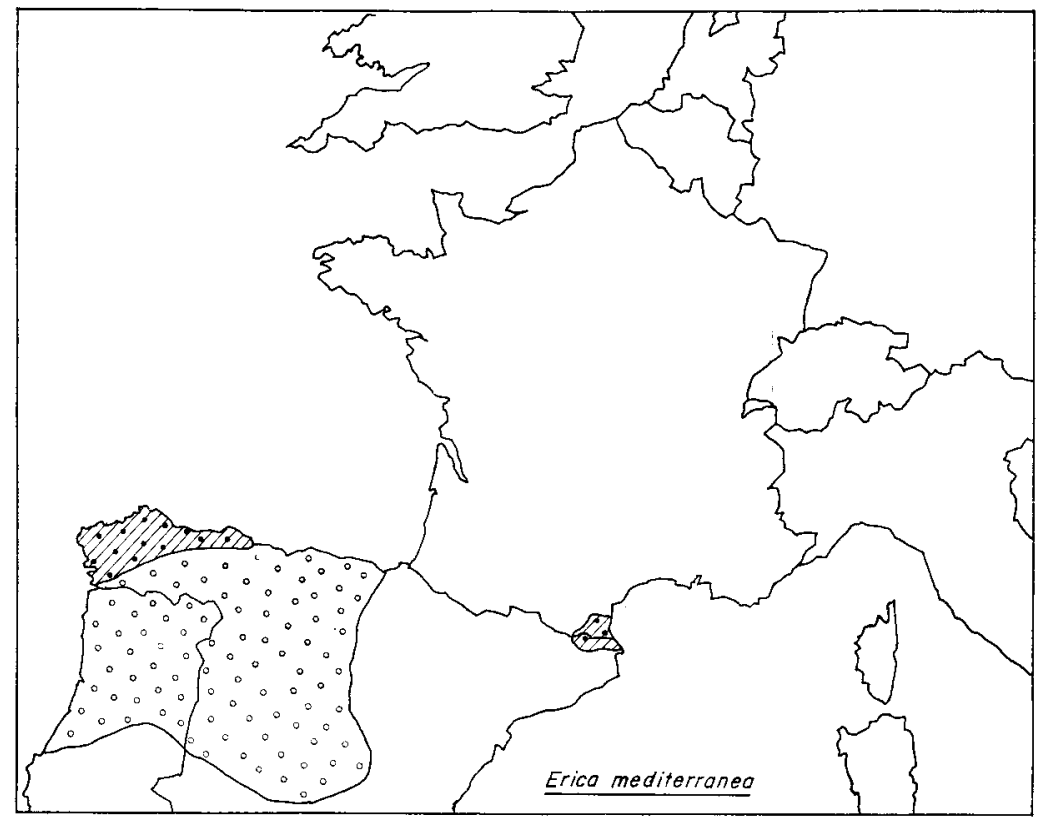

FIG. 17 .

Le développement optimum des Éricacées de 1'Europe occidentale coïncide presque toujours avec un $\mathrm{pH}$ du sol compris entre 3,8 et 6 (J. Giroux, I948). Les Éricacées se présentent donc, en général, comme des individus acidophiles; elles ne paraissent calcifuges qu'en raison de l'alcalinité constante des terrains calcaires.

De très rares espèces trouvent sur le calcaire des conditions de végétation suffisantes à leur existence, Erica carnea, Arbutus alpina, Erica scoparia, dans certains cas Rhododendron hirsutum et surtout Erica multiflora - type méditerranéen en sont les meilleurs exemples.

Les Éricacées peuplent les milieux les plus divers : il s'agit en général de sousarbrisseaux atteignant quelques décimètres rarement plus, que 1'on peut rencontrer dans les landes, les bois, les marais tourbeux, les talus, les buissons. Notons que quelques espèces : Erica mediterranea, Rhododendron ferrugineum peuvent atteindre, voire dépasser, I $\mathrm{m}$ de haut, tandis qu'Erica arborea, Arbutus unedo pouvant atteindre 3 à $4 \mathrm{~m}$ de haut constituent de vrais arbres. 


\section{Répartition des Éricacées en altitude}

C'est sous forme de landes - au moins en France et pour les espèces de basse altitude — que sont rencontrées beaucoup d'Éricacées : il s'agit de peuplements très uniformes caractérisés par la prédominance de sous-arbrisseaux supportés le plus souvent par un sol argileux ou acide : tel est le cas des étendues de Calluna vulgaris SALISB., Erica scoparia L., Erica vagans I., Erica ciliaris I. du Sud-Ouest français.

S'opposant à ces espèces de basse et moyenne altitude (localisation comprise entre o et quelques centaines de mètres), d'autres atteignent et dépassent la zone des sapins dans les régions montagneuses, abondantes encore dans la zone alpine supérieure (2 500-3 ooo m d'altitude environ). Tel est le cas d'Erica carnea L., Rhododendron hirsutum L., Rhododendron ferrugineum L., Loiseleuria procumbens DESV., des différents Vaccinium, d'Oxycoccos PURsh., d'Arbutus uva-ursi L. et A. alpina L.

Il est donc possible de retrouver en France à des altitudes très variables, au moins un représentant de la famille.

Les différentes espèces d'Éricacées peuvent donc schématiquement du point de vue de leut répartition en altitude être groupées en :

a) Espèces de moyennes et fortes altitudes :

- Vaccinium vitis idaea L. pouvant atteindre $2800 \mathrm{~m}$ dans les Alpes, I $300 \mathrm{~m}$ dans les Vosges et le Jura.

- Vaccinium uliginosum L. pouvant atteindre $3000 \mathrm{~m}$ dans les Alpes, I ooo m à I $300 \mathrm{~m}$ dans le Jura.

- Vaccinium myrtillus L. pouvant atteindre $2500 \mathrm{~m}$ dans les Alpes, I $800 \mathrm{~m}$ dans les Pyrénées.

- Oxycoccos vulgaris PURsh. que l'on peut rencontrer jusqu'à $2000 \mathrm{~m}$ dans les Alpes, I $300 \mathrm{~m}$ dans le Jura.

- Arbutus alpina L. que l'on peut rencontrer jusqu'à 2500 dans les Alpes.

- Arbutus uva-ursi L. que l'on peut rencontrer jusqu'à $2200 \mathrm{~m}$ dans les Alpes, 700 à I $000 \mathrm{~m}$ dans le Massif central.

- Rhododendron fermgineum L., Rhododendron hirsutum L., Erica carnea L. peuvent dépasser $2000 \mathrm{~m}$ dans les Alpes.

- Doboecia polifolia DON., Loisebeuria procumbens DESv., Phyllodoce cerulea FRIES. peuvent également atteindre $2000 \mathrm{~m}$ et même $2500 \mathrm{~m}$.

b) Espèces de faible altitude:

Erica vagans L., Erica tetralix L., Erica ciliaris L., Erica lusitanica RUD., Erica scoparia L., Erica cinerea L., Erica arborea L. pour la plupart rencontrées dans l'ouest, le sud-ouest ou le sud de la France, ne s'élèvent pas lorsqu'elles atteignent les zones montagneuses à plus de quelques centaines de mètres.

Remarquons, enfin, que Calluna vulgaris SALISB. est capable de s'adapter à des altitudes très diverses, retrouvée en peuplements massifs dans les landes du SudOuest au niveau de la mer, la même plante apparaît encore à des altitudes de 1'ordre de $2500 \mathrm{~m}$ dans les Alpes suisses. 


\section{TABI,EAU I}

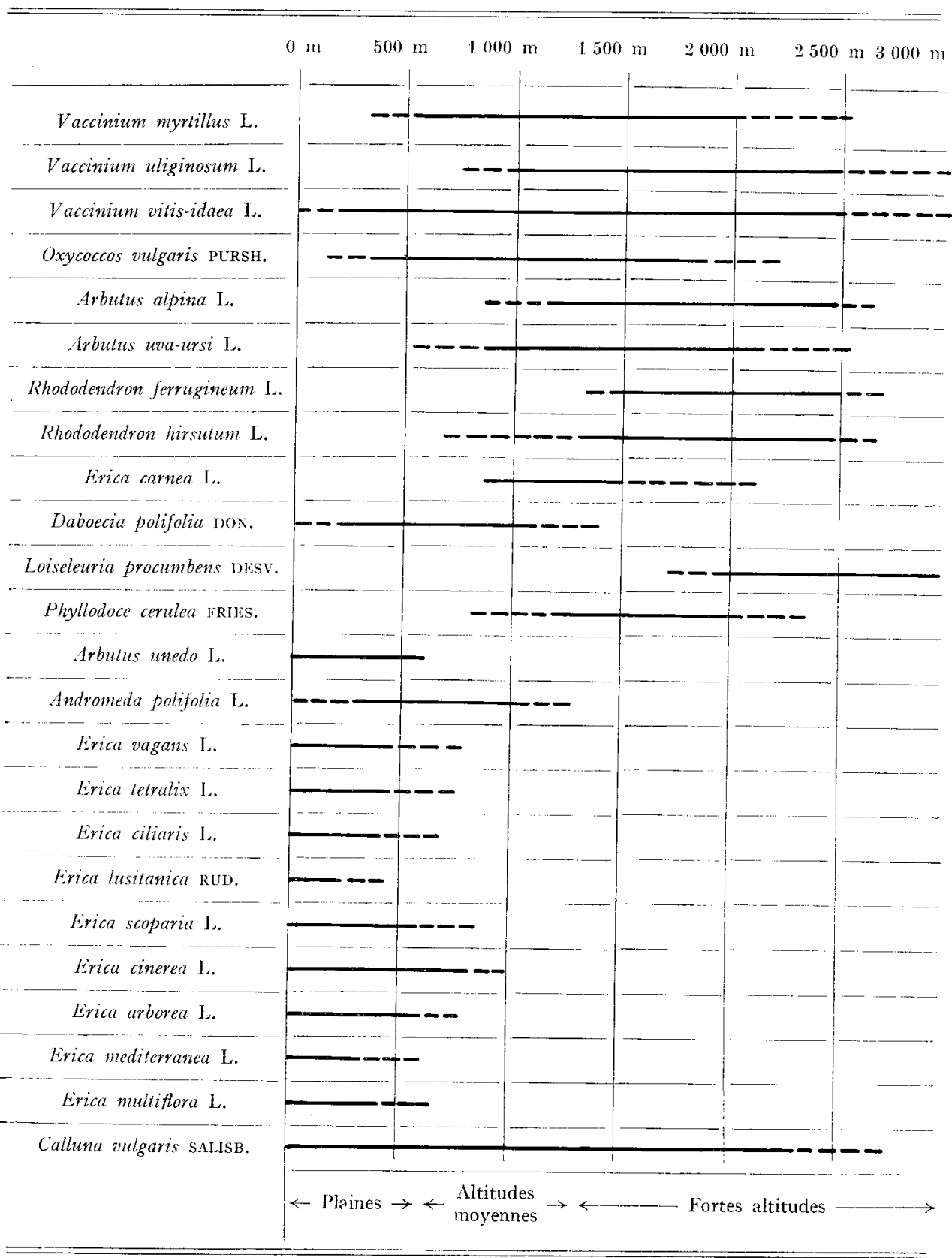


TABLEAU 2

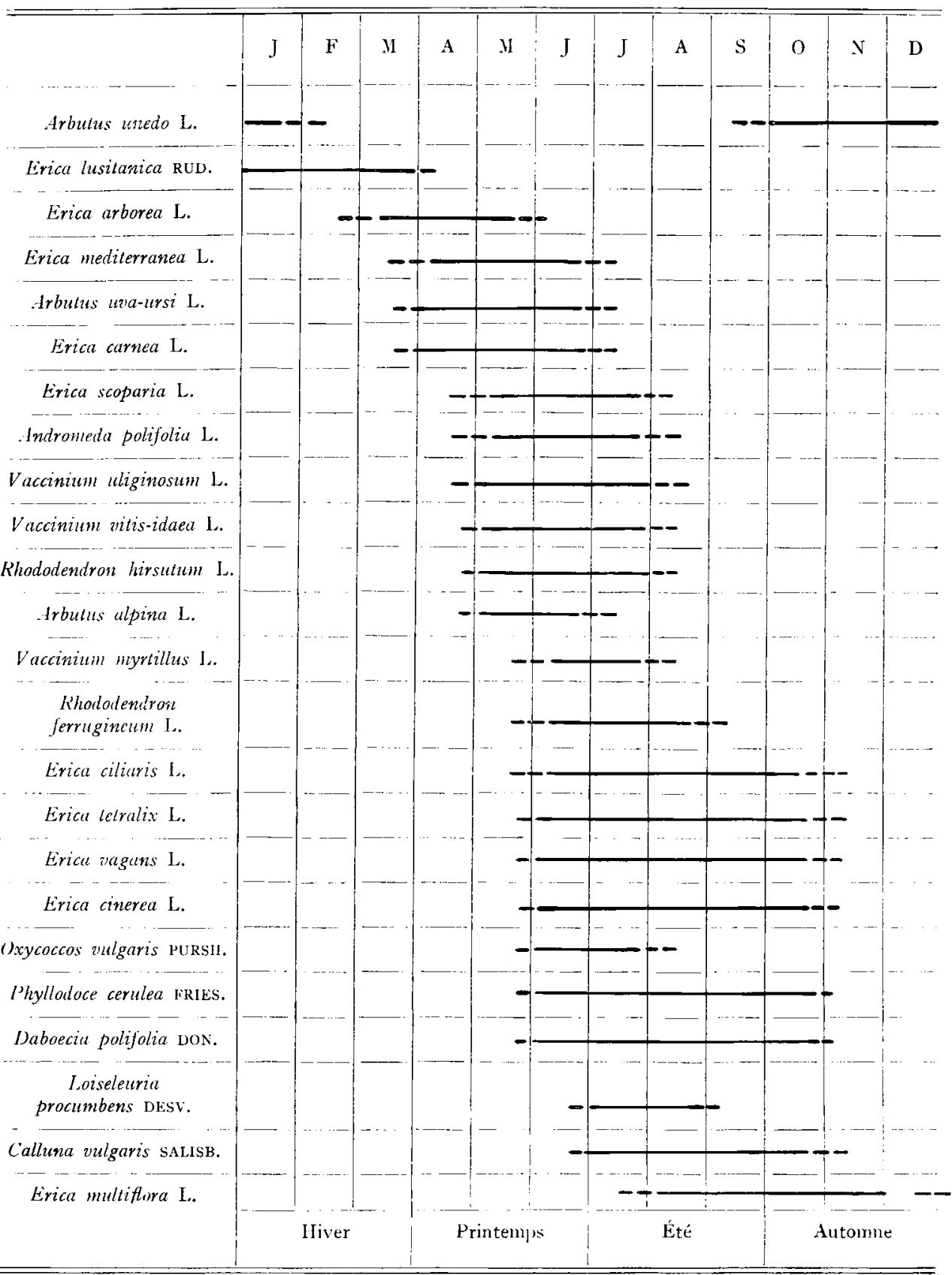


Ces répartitions en altitude peuvent être schématiquement regroupées sous forme d'un tableau (voir tabl. I).

Nous avons de même, sous forme de tableau (tab1. 2), réuni les époques defloraison des différentes espèces d'Éricacées acclimatées en France. Il est aisé de constater que pendant une bonne partie de l'année, les Éricacées constituent pour l'Abeille une source importante de nectar et de pollen.

\section{III. - LES ÉRICACÉES DANS LES MIELS FRANÇAIS}

\section{A. - MATÉRIEL ET MÉTHODES}

2334 préparations mélissologiques - collection du laboratoire, ont été mises à notre disposition ; du fait de l'origine parfois inconnue ou incertaine d'une partie de celles-ci, I 367 échantillons seulement ont été retenus dont la provenance est bien connue.

('es préparations ont été examinées sous le microscope.

Ians le cas où elles ne renfermaient pas de pollen d'Ĺricacées, seule la nature du pollen dominant était notée. (Rappelons que ZANDER (I935) a défini un pollen comme étant dominant lorsque ce pollen est rencontré au moins 45 fois sur too. Le même auteur parle de pollens d'accompagnement lorsque ce taux est compris entre I5 p. I00 et 45 p. I00; enfin, de pollen isolé si le taux est inférieur à 15 p. IOO).

Dans le cas de préparations contenant des pollens d'Éricacées, nous avons noté : la nature du pollen dominant, le nombre de grains provenant d'Éricacées rencontrés sur 500 grains comptés (ce nombre de 500 ayant été calculé par Ph. VERGERON (I964) comme étant un minimum nécessaire, conciliable avec la précision requise par la suite dans les évaluations statistiques), les différentes Éricacées présentes, leurs proportions respectives enfin ont été notées.

Après examen des I 367 préparations retenues, nous avons regroupé les fiches correspondantes en régions géographiques d'origine : c'est alors que s'est posé le problème du découpage de la France en régions géographiques aussi naturelles que possible.

Le découpage réalisé par l'Institut national de la Statistique et publié dans l'ouvrage : Régions géographiques de la France nous a semblé convenir à notre travail (fig. 18). mitée.

De nouvelles fiches ont été alors établies : une par région géographique précédeınment déli-

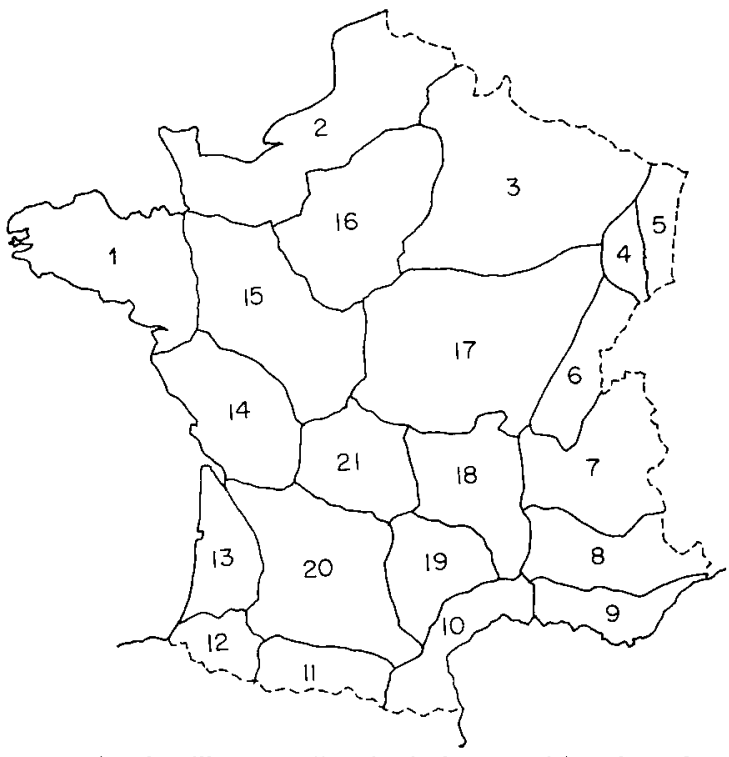

18. Découpagre régrional utilisé pour l'étude de la répartition des échantillons de miel 
Nous reproduisons, ici, à titre d'exemple, celle concernant la région $\mathrm{n}^{\circ} \mathrm{ro}$, région groupant approximativement Languedoc, Roussillon, Pyrénées méditerranéennes ; I07 échantillons la concernent. Parmi ces 107 préparations 79 renferment des pollens d'Éricacées, 28 en sont dépourvues.

La répartition des différentes espèces est la suivante :
Erica vagans L. retrouvé 29 fois, soit approximativement dans 29 p. Ioo des cas.
Rhododendron ferrugineum L. : 7 fois, soit approximativement dans 7 p. roo des cas.
Calluna vulgaris SALISB. : $5^{\text {I }}$ fois, soit approximativement dans $5^{\circ} \mathrm{p}$. 100 des cas.
Vaccinium myrtillus $L .: 9$ fois, soit approximativement dans 9 p. roo des cas.
Erica arborea $L . .: 46$ fois, soit approximativement dans 45 p. 100 des cas.
Erica cinerea $L .: 3^{I}$ fois, soit approximativement dans 31 p. roo des cas.
Erica multiflora L. : 3 fois, soit approximativement dans 3 p. roo des cas.

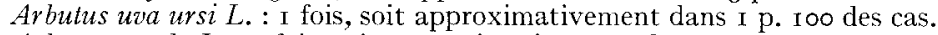
Arbutus unedo L. : 5 fois, soit approximativement dans 5 p. Ioo des cas.

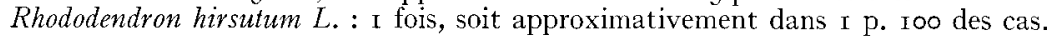

Les pourcentages nous indiquent, pour une région donnée, la fréquence d'apparition d'échantillons de l'espèce considérée : cette fréquence d'apparition a été arbitrairement définie :

Ont été déclarées très rares, les espèces rencontrées dans moins de 5 préparations sur ıoo, rares ou peu fréquentes celles apparaissant dans 5 à $20 \mathrm{p}$. 100 des préparations. Les espèces rencontrées dans 20 à $40 \mathrm{p}$. 100 des préparations ont été classées courantes; au-delà de ce pourcentage, nous avons parlé d'espèces très courantes ou très fréquentes.

Cette notion de fréquence d'apparition, définie par le pourcentage de préparations dans lesquelles se retrouve le pollen considéré, dans une région donnée, est donc bien différente de celle de mode d'apparition qui, nous l'avons vu, est basée sur le pourcentage de rencontre du pollen en question à l'intérieur de la préparation considérée.

\section{B. - RÉsultats}

Sous forme de tableaux (tabl. 3 et 4 ), nous avons groupé les fréquences et les modes d'apparition des différentes espèces d'Éricacées à l'intérieur de chacune des régions géographiques précédemment définies. Ces résultats nous ont permis de superposer aux cartes de distribution géographique, les aires de distribution mélissologiques; l'étude des concordances, des rapports plus ou moins étroits entre les deux types d'aires fera 1'objet du prochain chapitre.

Nous signalerons également que, jusqu'à maintenant, les analyses mélissopalynologiques n'ont fait mention, en ce qui concerne les Éricacées de France que de peu d'espèces : Calluna vulgaris SAI,ISB., Arbutus unedo L., Erica vagans L., Erica cinerea L., Erica arborea L. L'étude réalisée nous a permis de montrer qu'en fait bon nombre d'Étricacées sont retrouvées dans les miels français : outre les espèces précédemment citées, il convient de mentionner : Rhododendron ferrugineum L., Rhododendron hirsutum L., Arbutus alpina L., Erica ciliaris L., Erica tetralix L., Erica carnea L., Erica multiflora L., Erica lusitanica RUD.

L'analyse mélissologique nous a permis de mettre en évidence I 6 des 26 espèces d'Éricacées décrites. Il s'agit de :

Rhododendron ferrugineum L.
Rhododendron hirsutum I.
Vaccinium myrtillus L.
Calluna vulgaris sALISE.
Arbutus unedo I.
Arbutus alpina L.
Arbutus uva-ursi L.
Erica vagans L.

Rhododendron ferrugineum L.

Vaccinium myrtillus L.

Calluna vulgaris SALISB.

Arbutus unedo L.

Arbutus uva-ursi L.

Erica vagans L.

\author{
Erica ciliaris I. \\ Erica arborea L. \\ Erica cinerea $\mathrm{L}$. \\ Erica carnea I. \\ Erica lusitanica RUD. \\ Erica mediterranea L. \\ Erica multiflora L. \\ Erica tetralix L.
}


TABLEAU 3

EREOUENCE D'APPARITYON DES DIFFERENTES ESPECES D'ERICACEES (en \%)

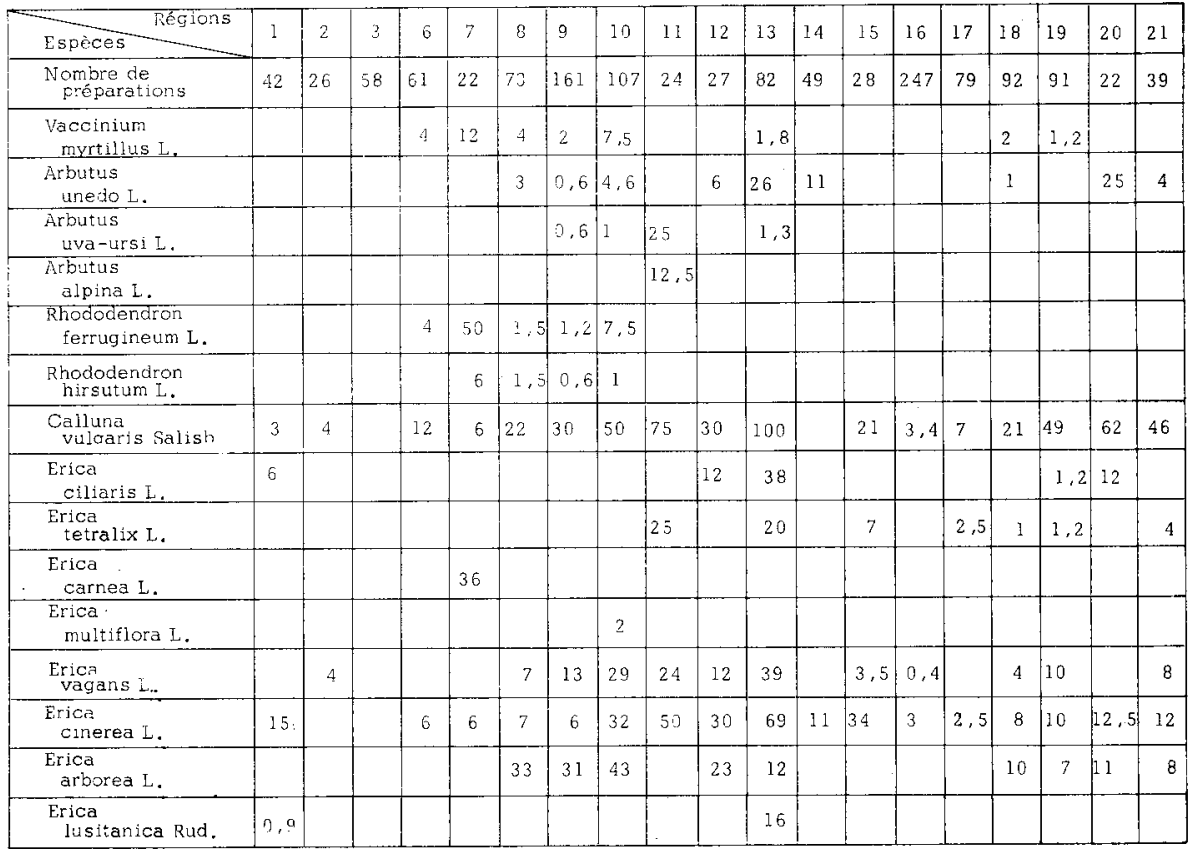

TABLEAU 4

MODE D'APPARITION DES DIFFERENTES ESPEECES D'ERICACEES

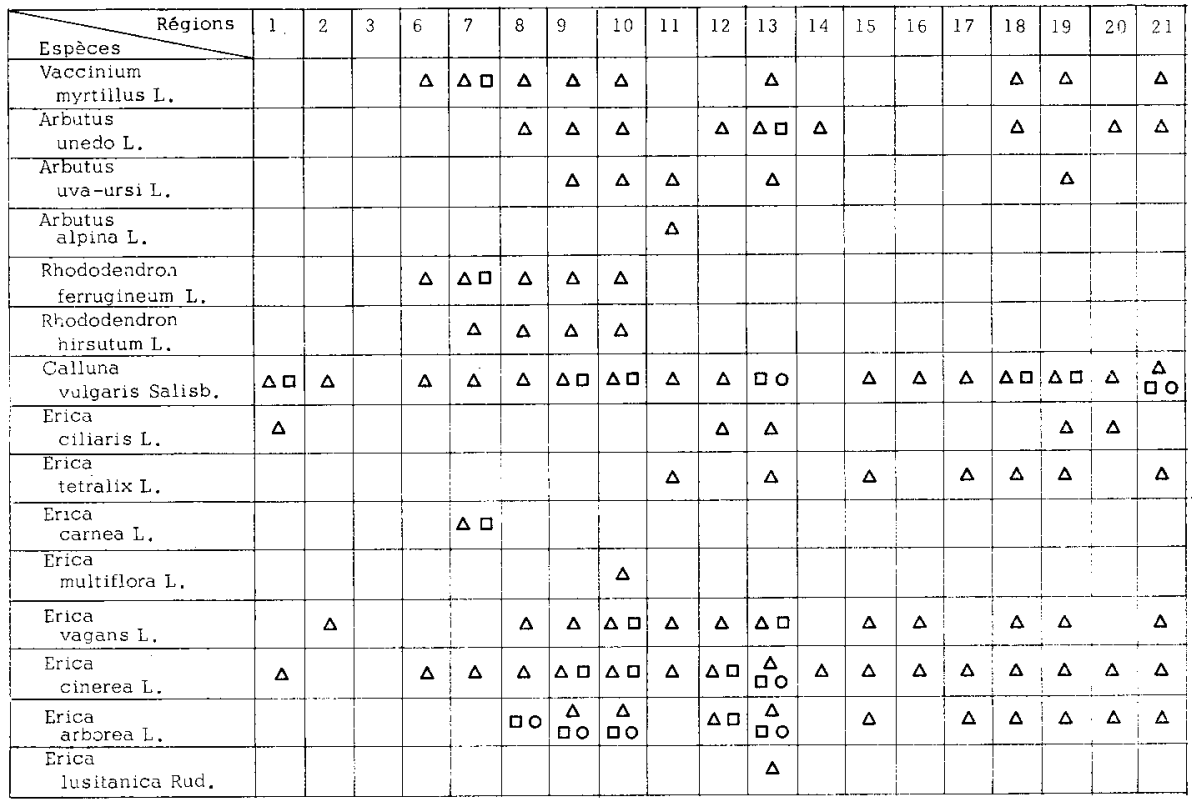


Cette étude n'a donc pas permis de retrouver :

Erica scoparia L.

Loiseleuria procumbens DESV.

Vaccinium uliginosum $\mathrm{L}$.

Vaccinium vitis-ideae L.

Phyllodoce cerulea FRIES.
Andromeda polifolia L.

Oxycoccos vulgaris PURsH.

Daboecia polifolia DON.

Erica umbellata GRAY.

En ce qui concerne Erica stricta DON. et Erica umbellata GRAY., ceci n'est pas surprenant : Erica stricta n'est mentionné qu'en Corse, Sardaigne, côte orientale de l'Espagne, or, parmi les échantillons mis à notre disposition, deux seulement proviennent de Corse, ce qui peut expliquer que le pollen correspondant n'ait pas été rencontré.

Par ailleurs Erica umbellata GRAY., n'ayant pas été à notre connaissance introduite en France, il semble normal que l'analyse mélissologique n'en fasse pas mention. Son pollen a été néanmoins étudié en raison du rôle capital qu'il joue dans les miels d'Espagne (L,OUVEAux, VFrgeron, I964). I1 était intéressant de vérifier son absence totale dans les miels français.

L'exiguité des aires de distribution géographique des genres Phyllodoce, Loiseleuria, Daboecia, Oxycoccos qui, par ailleurs, ne constituent pas de peuplements massifs là où ils se trouvent, explique que les pollens correspondants n'aient pas été retrouvés dans les miels.

La faiblesse du nombre d'échantillons concernant les régions ardennaise, alsacienne, lorraine, vosgienne, jurassienne et alpine septentrionale peut expliquer que les pollens d'Andromeda polifolia L. d'une part, des Vaccinium vitis-ideae L. et Vaccinium uliginosum L. d'autre part, espèces par ailleurs peu abondantes là où elles existent, n'aient pas été décelés à l'examen mélissologique.

Le cas d'Erica scoparia L., par contre, surprend davantage, la plante couvre d'immenses étendues et pourtant elle n'est pas visitée par 1'Abeille, contrairement à ce qu'avait pensé $\mathrm{G}$. BONNIER : deux hypothèses peuvent être avancées, ou bien le nectar produit est trop dilué, il n'attire pas l'abeille, ou bien il est inaccessible et l'insecte ne peut évidemment le prélever; peut-être aussi ce nectar est-il produit en trop faible quantité, ce cas mériterait d'être plus précisément étudié.

En résumé, ce dernier point mis à part, le cas de toutes les autres Éricacées dont le pollen n'a pu être retrouvé à l'examen mélissologique peut vraisemblablement s'expliquer par :

- 1'exiguïté de leur aire de distribution géographique, ou,

- la faiblesse du nombre d'échantillons concernant les régions intéressées.

Quoi qu'il en soit, les Éricacées représentent en France une partie importante de la flore mellifère : elles constituent pour l'Abeille une source très abondante de nectar et de pollen :

Les Rhododendrons, par exemple, en peuplements denses dans les Alpes, constituent au-delà de la zone des sapins une zone dite des Rhododendrons (I 500-2 $500 \mathrm{~m}$ environ), ils représentent de mai à août dans ces zones d'altitude d'excellentes sources de nectar.

Erica carnea L., dans les mêmes zones que précédemment, constitue, dès le début du printemps, une source importante de nectar et de pollen. 
Il en va de même d'Erica tetralix L., d'Erica vagans L., d'Erica cinerea I., d'Erica ciliaris L. qui, de juin à octobre, fournissent à l'Abeille nectar et pollen dans les régions côtières atlantiques de l'ouest de l'Europe.

Nous n'oublierons pas enfin le cas de Calluna vulgaris, 1'une des plantes mellifères les plus importantes de la fin de l'été en Europe et qui fournit chaque année dans de vastes régions une récolte abondante d'un miel à peu près pur.

\section{IV. - RAPPORTS ENTRE DISTRIBUTION GÉOGRAPHIQUE E'T DISTRIBUTION MÉLISSOLOGIQUE DES ÉRICACÉES}

\section{A. ÉTUdE DE IA CONCORDANCE DES DEUX TYPES D'AIRES}

Nous nous proposons de voir dans quelle mesure l'aire de distribution mélissologique d'une espèce donnée s'accorde avec celle de distribution géographique. La première, peut-elle confirmer, infirmer, compléter la seconde?

Les cartes de distribution géographique (fig. 2 à $\mathrm{I} 7$ ) ont alors été reprises et complétées grâce aux données recueillies lors de l'analyse pollinique des échantillons de miel disponibles.

Nous attirerons ici l'attention du lecteur sur la notion de valeur toute relative des limites figurées : les aires géographiques indiquées n'ont de valeur que considérées dans leur ensemble : ces aires sont globales et donc en conséquence schématiques ; ces cartes représentent un premier travail d'approche pouvant être considéré comme une méthode pour un travail ultérieur.

Il faut se souvenir par ailleurs que le tracé des aires de distribution mélissologique a pu être biaisé par plusieurs facteurs difficilement contrôlables : transhumance possible de certaines ruches et incertitude concernant l'origine très précise d'un certain nombre d'échantillons de miel ; facteurs auxquels il faut ajouter le fait que le nombre de préparations mélissologiques dont nous disposons ne nous a pas permis de "couvrir " significativement l'ensemble du territoire français.

Ainsi, peuvent s'expliquer d'une part le fait que pour certaines espèces, les aires de distribution géographique apparaissent plus restreintes que les aires de distribution mélissologique correspondantes, et d'autre part la présence a priori anormale dans certains miels, de pollens étrangers à la région, ceci du fait de la transhumance éventuelle des ruches. Ceci dit, voyons pour chaque espèce décrite, comment s'accordent les deux types d'aires.

I. Vaccinium myrtillus I. : les deux types d'aire s'accordent, néanmoins la myrtille ne paraît être butinée que dans la partie méridionale de son aire de distribution géographique.

2. Arbutus unedo L. : la concordance des deux aires est rigoureuse, l'arbousier apparaît butiné sur l'ensemble de son aire de répartition et plus spécialement dans la partie occidentale de cette aire.

3. Arbutus $u v a-u r s i$ L. : le pollen n'a été retrouvé qu'en très faible quantité, la présence de ce pollen dans un miel landais résulte vraisemblablement de la culture de la plante en tant que plante ornementale. 
4 et 5 . Rhododendron ferrugineum L. et Rhododendron hirsutum L. apparaissent butinés sur l'ensemble de leur aire de distribution géographique.

6. Erica ciliaris I. : semble butinée sur une grande partie de son aire de distribution géographique, plus particulièrement dans la partie méridionale.

7. Erica tetralix I. : par contre, ne semble pas attirer spécialement l'Abeille: la plante n'est visitée que sur une partie restreinte de sa zone géographique.

8-9. Evica carnea L. n'a été retrouvée que dans des miels provenant de HauteSavoie; Erica multiflora L. ne fut mise en évidence que dans des échantillons de l'Hérault : les deux types d'aires concordent ici parfaitement.

Io. Erica vagans L. : est butinée sur 1'ensemble de son aire de distribution géographique mais de façon très accessoire au Nord d'une ligne Bordeaux-Toulon, alors que la plante semble être activement recherchée par l'Abeille au sud de cette limite ; un " trou » toutefois étant à noter dans la région du Tarn-et-Garonne, peut-être la plante dans cette région est-elle plus rare. Notons que, la présence en quantité notable de pollen d'Erica vagans, dans certains miels landais, est selon tonte vraisemblance imputable à la pratique de transhumance des ruches, courante dans cette région.

Ir. Le cas de Calluna vulgaris sAIISB. est un peu particulier; abondante sur l'ensemble du territoire français, la plante ne semble activement visitée par l'Abeille que dans les Landes, les régions de l'Aveyron et du Limousin — régions productrices de miels dits " miels de Callune " - la plante est cependant visitée, à plus faible échelle, un peu partout, le nord du pays et la région vendéenne faisant exception.

12. Le cas d'Erica cinerea I. est presque calqué sur celui de Calluna vulgaris SALISB. : la plante est abondante un peu partout en France, son pollen se retrouve également dans les miels de nombreuses régions, mais n'est présent à l'état dominant que dans les miels du Sud-Ouest français; une sorte de discrimination là encore semble s'exercer sans que la cause en soit connue.

13. Erica arborea L. butinée par l'insecte partout où elle existe constitue un auxiliaire précieux lorsqu'il s'agit de déterminer l'origine d'un miel. Dominant dans de nombreux miels provenant du littoral méditerranéen, il n'apparaît partout ailleurs qu'à l'état isolé.

I4 et I5 : Deux autres Ericacées enfin sont utiles au mélissopalynologiste :

- Erica lusitanica RÜD. assez peu visitée par 1'Abeille mais ceci de façon très localisée :

- Erica umbellata GRAY. très commune dans les miels espagnols, jamais rencontrée dans les miels français et dont la présence, de ce fait, permet de conclure à une origine non française du produit.

Nous pouvons schématiquement résumer ces constatations :

- Vaccinium myrtillus L. ) sont surtout visitées dans la partie méridionale de Erica tetralix L. leur aire de distribution géographique. 
- Evica cinerea L. intéresse plus particulièrement 1'Abeille dans la partie Ouest de son aire.

- Erica arborea L. apparaît butinée partout où elle existe, même là où elle est assez rare.

- Calluna vulgaris SAIISB. constitue pour l'Abeille une plante mellifère de premier choix, sauf dans la partie nord-est de son aire géographique.

Toutes les autres Éricacées mellifères: Arbutus unedo L., Arbutus uva-ursi L., Arbutus alpina L., Erica lusitanica RUD., Erica carnea L., Erica multiflora L., Erica vagans L., Rhododendron ferrugineum L. et Rhododendron hirsutum $\mathrm{L}_{1}$. sont visitées par l'insecte sur l'ensemble de leur aire de distribution géographique.

Il est maintenant intéressant de chercher à voir quelles espèces sont plus particulièrement utiles au mélissopalynologiste lorsqu'il doit déterminer l'origine géographique d'un miel.

\section{B. - Possibilités de dÉtERmination DE I'ORIGINE DES MIELS}

I. Considérées isolément, les espèces suivantes permettent d'attribuer à l'échantillon :

a) une origine montagneuse : Erica carnea L., Rhododendron hirsutum L. (Alpes du Nord) ; Rhododendron ferrugineum L. (Alpes du Nord, Corbières, Pyrénées médit.).

b) Une origine atlantique (plus particulièrement région du Sud-Ouest français) : Erica ciliaris L., Evica tetralix L.

c) La présence d'Erica multiflora L. laisse à penser que le miel est d'origine languedocienne.

En ce qui concerne les autres espèces mellifères, du fait de la plus grande extension de leur aire géographique, l'origine du miel qui les contient est plus difficile à déduire.

Arbutus unedo L. se retrouve dans les miels méditerranéens aussi bien qu'atlantiques ne dominant cependant que dans les miels landais.

De même Erica cinerea L., Calluna vulgaris SAIısB. très fréquemment rencontrés dans les miels ne sont pas, à cause de l'importance de leur aire de butinage, de bons auxiliaires du mélissopalynologiste ; sauf s'ils sont retrouvés à l'état dominant, auquel cas ils augurent d'une origine landaise pour le premier, landaise, limousine ou aveyronnaise pour les seconds.

Erica arborea L. peut laisser prévoir une origine méditerranéenne de l'échantillon.

Erica lusitanica RUD. bien que rarement rencontrée est le critère sûr d'une origine littorale landaise ou bretonne.

Il va de soi que ces orientations suggérées par la présence de telle ou telle Éricacée doivent être confirmées, parfois infirmées, par l'examen du spectre pollinique tout entier.

2. Considérés du point de vue de leur association dans un spectre mellifique, les pollens d'Éticacées deviennent alors de très précieux guides pour le mélissopalynologiste. 
Une association $E$. arborea $\mathrm{L}_{\text {., }} E$. vagans $\mathrm{L}_{\text {., }}, E$. multiflora L., accompagnée

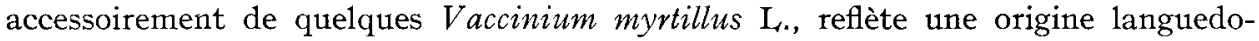
cienne du miel considéré.

- Un spectre typiquement landais pourra montrer réunis les pollens : d'Erica ciliaris L., d'Erica tetralix L. auxquels peuvent se joindre quelques Arbutus unedo L. et bien sûr en grande quantité le pollen de Calluna vulgaris SAIISB.

Un autre spectre landais pourra renfermer Calluna vulgaris SALISB., Erica cinerea L., Erica ciliaris L., accessoirement Erica vagans L.

De nombreuses combinaisons de ces pollens sont évidemment possibles.

- Parmi les spectres d'altitude, Erica carnea L. se retrouvera associé à Rhododendron ferrugineum $\mathrm{L}_{\text {. }}$ dans les miels des Alpes du Nord impliquant une altitude de l'ordre de I 500 à $2000 \mathrm{~m}$ ou plus.

- Dans les zones élevées des Corbières, pourront être réunis, en faible quantité, es pollens de Rhododendron ferrugineum L., Arbutus uva-ursi L., voire quelques Vaccinium myrtillus L., la présence de Calluna vulgaris saLISB. étant bien entendu possible.

- En Bretagne, nous pourrons rencontrer E. cinerea L., E. ciliaris L., Calluna mulgaris SALISB.

- Dans une zone géographique, plus centrale - Limousin, Marche, Berry, Touraine, il est possible de retrouver associés les pollens de Calluna vulgaris SAI,Iss., E. letralix L., E. cinerea L., éventuellement d'E. vagans $\mathrm{L}$.

Enfin, une association d'Erica arborea I., Erica vagans L. auxquels peuvent se joindre quelques Arbutus unedo L. ou Vaccinium myrtillus L., témoigne d'une origine littorale méditerranéenne du miel.

Ici, comme précédemment, il est certain que l'examen du spectre mélissologique tout entier et non limité aux Éricacées, complétera les données nécessaires à la détermination de l'origine du miel.

Ce travail a été fait pour quelques miels, nous allons maintenant aborder cette question.

\section{C. - ASSOCIATIONS POLIINIQUES DANS LESQUEILES APPARAISSENT LES ÉRICACÉES}

Dans ce but, des analyses polliniques complètes ont été réalisées. Portant sur 22 échantillons (provenant de diverses régions géographiques et caractérisés par le fait que tous ont pour pollen dominant un pollen d'Ericacées) ces analyses ont permis de mettre en évidence et d'étudier les associations polliniques rencontrées dans une région géographique donnée. (tableau 5)

- Les quatre premiers spectres typiquement landais sont remarquables par le faible nombre d'espèces présentes, pauvreté qui reflète bien celle de la flore locale. Éricacées abondantes accompagnées d'un faible nombre d'espèces présentes en quantité importante : Jasione montana L., Hedera helix L., Rubus Rhamnus frangula L., Ulex; il s'agit là d'un ensemble bien caractéristique du sous-bois landais.

- Les miels de Callune de l'Aveyron, par contre, sont beaucoup plus riches et caractérisés par la présence d'un bon nombre de pollens typiques des prairies de moyenne altitude : Trifolium, Onobrychis, Helianthemum, Centaurea cyanus L., Cen- 
TABLEAU 5

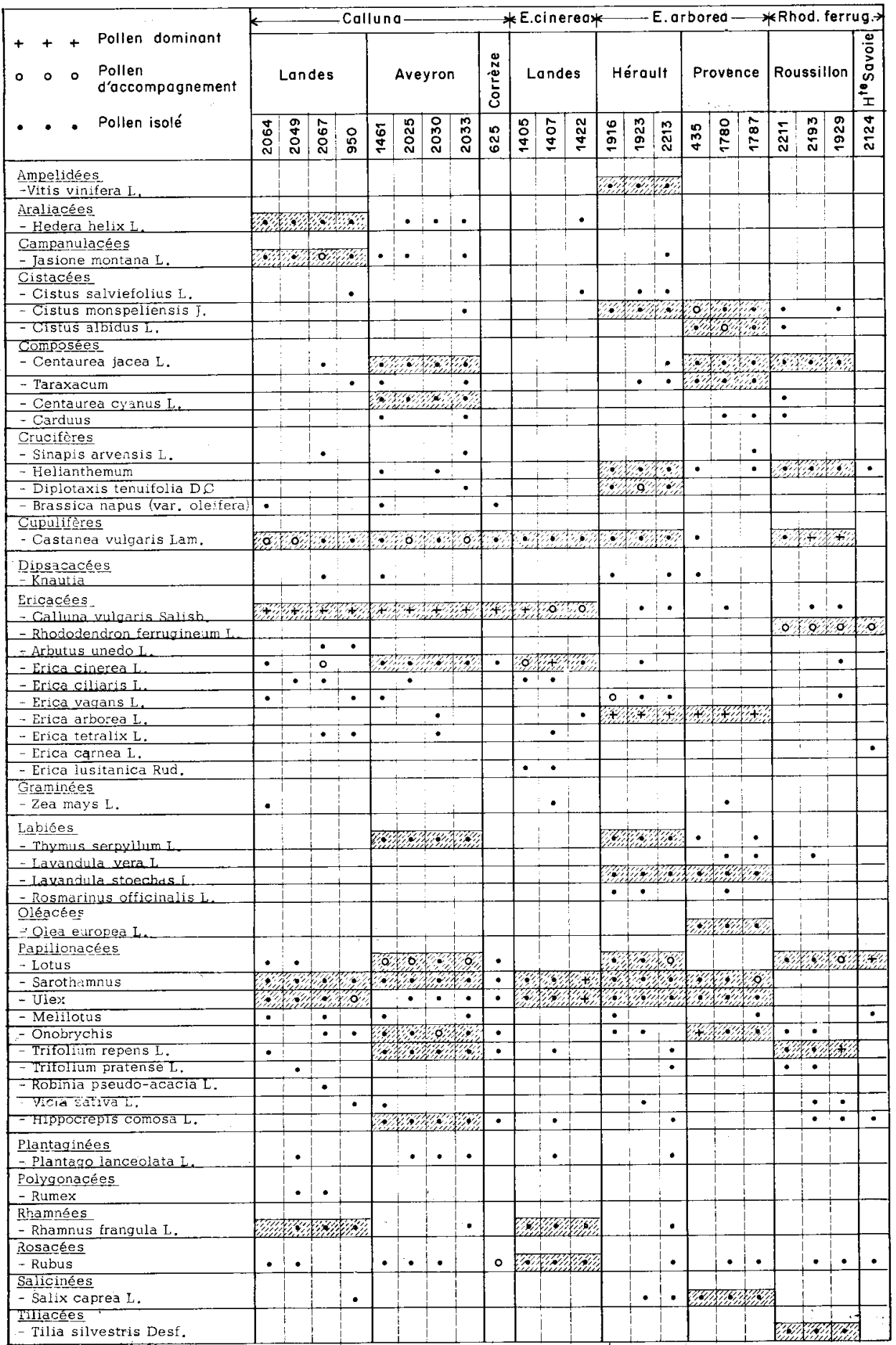


taurea jacea L. La présence de Thymus, par ailleurs, traduit une origine déjà méridionale.

- Le spectre mélissopalynologique se rapportant à la région du plateau de Millevaches (Corrèze) montre, comme dans les cas précédents, des pollens de plantes caractéristiques de prairies de moyenne altitude ; la Callune y est encore dominante, mais les espèces présentes sont moins nombreuses et une tendance plus septentrionale se fait sentir et se traduit par l'absence de Thymus par exemple.

- Les miels de l'Hérault, choisis parmi ceux où Erica arborea I. représentent le pollen dominant, sont caractérisés par la présence de plantes à incidence nettement méditerranéenne : Evica vagans L., Cistacées, Vitis, Thymus, Lavandula; à ces espèces, se joignent Rubus, Lotus, Ulex traduisant l'existence de prairies de moyenne altitude.

- Dans les miels de Provence, outre les espèces méditerranéennes précédemment citées, il faut mentionner la présence à peu près constante du pollen d'Olea europea, plante bien caractéristique de cette région.

- Dans les zones d'altitude du Roussillon, le pollen de Rhododendron ferrugineum apparait. Les pollens de Tilia sylvestris DESF., Rubus, Trifolium repens L., Castanea vulgaris, espèces qui toutes atteignent et peuvent dépasser des altitudes de l'ordre de $\mathrm{I} 000$ à I $500 \mathrm{~m}$ dans les zones montagneuses des Corbières et des Pyrénées, confirment cette origine; la présence de Thymus traduit ici encore l'origine méridionale du miel.

- Enfin, Erica carnea L. est retrouvée associée à Rhododendron ferrugineum L. dans certains miels savoyards; la provenance du miel, ici, ne fait aucun doute: il s'agit de zones montagneuses et d'altitude déjà importante : $2000 \mathrm{~m}$ ou plus.

$A$ noter également la faiblesse dans ces régions du nombre d'espèces mellifères.

\section{CONCL,USION}

L'importance des Éricacées en tant que plantes mellifères n'est plus aujourd'hui à établir. Nous insisterons cependant sur le fait que peu d'espèces - les plus couramment et abondamment visitées il est vrai - étaient jusqu'à présent reconnues lors des analyses mélissopalynologiques, il s'agit de Calluna vulgaris SALISB., Arbutus unedo L., Erica cinerea L., Erica vagans I., Erica arborea L.

Il s'avère, en fait, que les deux-tiers environ des vingt-six espèces d'Éricacées d'Europe Occidentale, constituent pour 1'Abeille, une source de nectar et de pollen non négligeable; nous avons donc affaire, ici, à une famille de plantes mellifères très importante. Il est en conséquence intéressant de savoir déterminer les différents pollens appartenant à cette famille et de pouvoir pousser la détermination de ces pollens jusqu'au niveau spécifique, en effet, étant donné la fréquence avec laquelle sont rencontrés les pollens d'Ericacées dans les miels et la dissemblance des zones de répartition géographique des diverses espèces, une détermination qui s'arrêterait au niveau de la famille ou du genre, n'apporterait que très peu - voire aucun renseignement quant à 1'origine du produit. 
En fait, un certain nombre d'É,ricacées ne jouent qu'un rôle secondaire en tant que plantes mellifères : Arbutus uva-ursi L., A. alpina L., Erica tetralix L., Erica ciliaris L., Erica carnea L., Erica multiflora L., Erica lusitanica RUD., le rôle capital étant tenu quantitativement au moins par les quatre ou cinq genres précédemment cités.

Il n'en reste pas moins, comme nous l'avons vu au cours de cette étude, que la détermination de pollens retrouvés parfois en faible ou très faible quantité (Rhododendron, Erica carnea L., Erica lusitanica RUD., Erica multiflora L., Erica umbellata GRAY.) permet dans certains cas de s'orienter avec précision ou d'exclure une origine possible du miel.

La distinction des différents genres d'Éricacées entre eux ne semble pas poser de problèmes ardus, par contre, la différenciation au niveau spécifique est parfois délicate : les pollens des différentes espèces du genre Vaccinium ou du genre Rhododendron par exemple, sont assez difficiles à distinguer, celui d'Erica ciliaris L. pouvant être également assez difficilement différenciable de celui d'Erica tetralix L. ou de Vaccinium myrtillus I.

Nous avons vu que le problème se simplifiait quelque peu dès lors qu'il n'était plus envisagé que sous son seul aspect mélissologique. Parmi les seize espèces d'Éricacées visitées par l'abeille, une dizaine jouent un rôle capital, ce qui explique que nous ayons pour elles essayé de mettre au point une clé de détermination restreinte.

Nous remarquerons enfin que 1'activité du mélissopalynologiste travaillant sur des miels d'origine bien déterminée peut lui permettre par l'analyse des spectres obtenus de reconstituer approximativement la flore mellifère de la région considérée. Aucun renseignement concernant la flore extra-phanérogamique ne pourra bien entendu être obtenu par ce moyen, mais il semble aujourd'hui possible que des données d'ordre écologique se rapportant aux seules espèces mellifères puissent être recueillies par ce moyen. L'analyse mélissopolynologique a le mérite de poser voire dans certains cas de reposer - un certain nombre de problèmes de type écologique et il ne semble pas exagéré de penser que l'Abeille puisse venir un jour en aide aux phytogéographes.

Rę̧u pour publication en novembre 1966.

\section{SUMMARY}

THE POLLEN OF THE " ERICACEAE " IN FRENCH HONEYS

For each of the ericaceous species considered, different measurements were carried out on twenty pollen grains. The ornementation of the pollen grains and its dimensions evaluated statistically constitute a good criterion for determination. The distinction is particularly delicate in the case of the species of the genera Vaccinium and Rhododendron. While retaining only the most decisive characteristics, it has been possible to develop a key for "fresh " ericaceous pollen. Many of the Ericacae form part of heathland or other very uniform populations, characterized by the predominance of sub-shrub and most frequently supported by a clay or acid soil. By means of pollen analysis of $13^{67}$ samples of french honey, it was possible to detect 16 to 26 species of the Ericacae From a study of the relationships between the geographical distribution and the melissological distribution of the Ericaceae, it can be noted that the melissopalinologist, working on honeys of well-defined origin, can, by analysis of the spectrum obtained, approximately reconstitute the melliferous flora of the region under consideration. Furthermore, the study of the ericaceous spectrum in a honey of unknown geographical origin leads to information that is generally very interesting as to the origin of the honey. 


\section{RÉFÉRENCES BIBLIOGRAPHIQUES}

Bonnier G., 1934. Flore complète illustrée en couleurs de France, Suisse, Belgique. 7, p. 54-64. Éd. Orlhac. Paris.

Coste H., I906. Flore descriptive el illustrée de la France. I, p. 503-513. Paris.

Dupont P., I962. La flore atlantique européenne, introduction à l'étude du secteur ibéro-atlanique et documents pour les cartes de producion végétale. Toulouse.

Fournier P., i946. Les quatre flores de France. P. Lechevalier, p. Paris, 699-705.

Gıroux J., 1934. Erica multiflora ou bruyères à heurs nombreuses. Thèse Montpellier, p. r6-22.

IIEGi. Illustrieste Flora von Milteleuropa.

IICLTEN E., 1950. Allas of the distribution of vascular plants in Nord-Wesl Europa. Stockholm.

Institut national de la Statistique et des Études économiques, s. d. Régions géographiques de la lirance, carte.

Jeanjean A. F., ig6r. Actes de la société linnéenne de Bordeaux. 99.

Louveaux J., VERgeron Ph., I964. Étude du spectre pollinique de quelques miels espagnols. Ann. Abeille, 1964, 7, 329-347.

Maurizio A., Louveaux J., I964. Pollen et Plantes mellifères d'Europe. V. Pollen et Spores, 1, 5-16. Oudfield F., I959. The pollen morphology of some of the west european Ericales. Pollen et Spores, 1, I9-48.

Van Campo M., Guinet Ph., ig6r. Les pollens composés. Pollen el Spores, 3 (2), 202.

VERGERON Ph., I964. Interprétation statistique des résultats et critères d'analyse pollinique des miels. Ann. Abeille, $7,349^{-364}$.

WillkoMм M., I.ANGE J., I87o. Prodromus Florae hispanicae seu synopsis methodica omnium plantarum in Hispania, sponte rascentium vel frequentius cultarum quae innotuerunt. Vol. II, Stuttgartiae. E. Schweizerbart, p. 335-348.

LANDER F., 1935. Bviträge sur Herkunflsbesimmung bei Honig. Pollengestallung und Herkunftsbestimmung bei Blütenhonig. Berlin. 